TRANSACTIONS OF THE

AMERICAN MATHEMATICAL SOCIETY

Volume 363, Number 8, August 2011, Pages 3977-4005

S 0002-9947(2011)05190-1

Article electronically published on March 16, 2011

\title{
SURFACES OF BOUNDED MEAN CURVATURE IN RIEMANNIAN MANIFOLDS
}

\author{
SIDDHARTHA GADGIL AND HARISH SESHADRI
}

\begin{abstract}
Consider a sequence of closed, orientable surfaces of fixed genus $g$ in a Riemannian manifold $M$ with uniform upper bounds on the norm of mean curvature and area. We show that on passing to a subsequence, we can choose parametrisations of the surfaces by inclusion maps from a fixed surface of the same genus so that the distance functions corresponding to the pullback metrics converge to a pseudo-metric and the inclusion maps converge to a Lipschitz map. We show further that the limiting pseudo-metric has fractal dimension two.

As a corollary, we obtain a purely geometric result. Namely, we show that bounds on the mean curvature, area and genus of a surface $F \subset M$, together with bounds on the geometry of $M$, give an upper bound on the diameter of $F$.

Our proof is modelled on Gromov's compactness theorem for $J$-holomorphic curves.
\end{abstract}

\section{INTRODUCTION}

The study of families of minimal surfaces and, more generally, constant mean curvature surfaces in Riemannian manifolds is a classical topic in differential geometry. Minimal surfaces in 3-manifolds, in particular, has received a lot of attention. In this paper, we focus our attention on closed (compact without boundary) surfaces in closed Riemannian $n$-manifolds with bounded mean curvature, generalising the case of surfaces of minimal surfaces (which are characterised by having mean curvature zero).

Our main result is a compactness result for surfaces of bounded mean curvature. Compactness results have played a central role in many areas of geometry. These include the Bers compactification of the moduli of curves, the compactification of the moduli of instantons leading to Donaldson's work on smooth four-manifolds and Gromov's compactness for the moduli of $J$-homolomorphic curves in symplectic manifolds, which is a central result in symplectic topology.

Received by the editors March 16, 2009.

2010 Mathematics Subject Classification. Primary 53C21.

This work was supported by the University Grants Commission (UGC). The second author was supported by DST Grant No. SR/S4/MS-283/05.

(C)2011 American Mathematical Society Reverts to public domain 28 years from publication 
Our result is closest in nature to the case of Riemannian manifolds with Ricci curvature bounded below. A fundamental result of Gromov is a compactness theorem, with sequences of such manifolds having subsequences converging to metric spaces.

In our case we consider smooth, embedded surfaces $F_{i}$ of a fixed topological type in a Riemannian manifold having bounded mean curvature and bounded area. We consider a fixed surface $F$ of the same topological type. By a parametrisation of $F_{i}$ we mean an appropriate choice of diffeomorphism from $F$ to $F_{i} \subset M$.

We show that, for a subsequence, we can find parametrisations so that the pullback metrics on $F$ converge to a continuous pseudo-metric on $F$ and the maps giving the parametrisations converge to a Lipschitz map.

The existence of such a limit has an immediate geometric consequence - we obtain an upper bound on the diameter of surfaces in a manifold with bounded mean curvature and area. Similar results have been obtained by P. Topping [11] for submanifolds of Euclidean space. In fact, as was pointed out to us by V. Tosatti, an alternative proof of our diameter bound can be obtained by combining Topping's theorem with the Nash embedding theorem.

For further applications, it is important to understand the limiting object, in particular to show that the pseudo-metric is not too degenerate. We show that the limiting pseudo-metric in fact has fractal dimension two. It would be very interesting to strengthen this to Hausdorff dimension, so that we have a metric and a measure as in the case of limits of Riemannian manifolds with Ricci curvature bounded below. Our result shows instead that there is a limiting capacity, i.e., a finitely additive measure.

Remark 1.1. It is easy to see that the upper bound on the area in our hypothesis is necessary. For instance, we can consider a sequence of geodesics $\gamma_{n}$ in the flat torus $S^{1} \times S^{1}$ with slopes converging to an irrational number. Then the products $\gamma_{n} \times S^{1} \subset S^{1} \times S^{1} \times S^{1}$ give totally geodesic (hence zero mean curvature) surfaces that are topologically tori. As the diameters of the corresponding pullback metrics tend to infinity, no subsequence can tend to a limit in our sense.

There is a large body of literature dealing with families of minimal surfaces in Riemannian 3-manifolds. The foundational work of W. Allard [1] deals with weak convergence of minimal surfaces in $n$-manifolds. For surfaces in 3 -manifolds, M. T. Anderson proved the following [2]: Let $\mathcal{M}_{n}$ denote the space of minimal embeddings of a closed surface of genus $\geq n$ in a complete 3 -manifold, endowed with the weak topology as a subset of the space of 2-varifolds. Then the boundary $\partial \mathcal{M}_{n}$ is contained in $\mathcal{M}_{\frac{n}{2}}$. As a corollary, it is shown that if the 3-manifold is compact and has negative sectional curvature, then $\mathcal{M}_{2}$ is compact.

For 3 -manifolds of positive curvature, H. Choi and R. Schoen prove 3$]$ the following compactness result: Let $N$ be a closed 3-manifold of positive Ricci curvature. Then the space of closed embedded minimal surfaces of fixed topological type, endowed with the $C^{k}$ topology, is compact if $k \geq 2$. In [12 $\mathrm{B}$. White generalized the result of Choi and Schoen to stationary points of arbitrary elliptic functionals defined on the space of embeddings of a compact surface in a 3-manifold, minimal surfaces being stationary points of the area functional. White's result is that compactness holds for such surfaces if we assume a bound on the area.

More recently, W. Minicozzi and T. Colding have studied [4] sequences of minimal surfaces in 3-manifolds of bounded genus without bounds on area. 


\section{Statement of Results}

We now state our results more precisely. Let $(M, g)$ be a closed, Riemannian $n$-manifold and let $H_{0} \geq 0$ and $A_{0}>0$ be fixed constants. Consider a sequence of closed, connected orientable surfaces $F_{j}$ in $M$ of a fixed genus $m$ with

(1) the norm of the mean curvature vector field bounded above by $H_{0}$ and

(2) area bounded above by $A_{0}$.

Let $i_{j}: F_{j} \rightarrow M$ denote the inclusion maps. Let $F$ be a smooth surface of genus $m$. We shall choose parametrisations of $F_{j}$ by $F$. It will be convenient for our constructions to regard these as diffeomorphisms $\varphi_{j}: F_{j} \rightarrow F-$ strictly speaking it is $\varphi_{j}^{-1}$ that gives a parametrisation of the surface $F$.

Given such parameters, the Riemannian metric on $M$ restricted to $F_{j}$ pulls back to Riemannian metrics $g_{j}=\left(i_{j} \circ \varphi_{j}^{-1}\right)^{*} g$ on $F$. We have corresponding metrics $d_{j}$ on $F$.

By a continuous pseudo-metric $d$ on $F$, we mean a continuous function $d: F \times$ $F \rightarrow[0, \infty)$ satisfying the triangle inequality, but not necessarily positivity. Our main result says that on passing to a subsequence, the distance functions converge to a pseudo-metric and the parametrisations to a Lipschitz map.

Theorem 2.1. There are homeomorphisms $\varphi_{j}: F_{j} \rightarrow F$ such that, after passing to a subsequence, the distance functions $d_{j}$ on $F$ corresponding to the pullback metrics $g_{j}=\left(i_{j} \circ \varphi_{j}^{-1}\right)^{*} g$ converge uniformly to a continuous pseudo-metric $d$ on $F$ and the maps $i_{j} \circ \varphi_{j}^{-1}$ converge in the $C^{0}$ topology to a Lipschitz map $i_{j}: F \rightarrow M$ (with respect to the pseudo-metric d).

As an application of Theorem 2.1 we obtain an upper bound on the intrinsic diameter of surfaces with bounded mean curvature and area in Riemannian manifolds. We note that P. Topping [1] has obtained upper bounds for the diameter of submanifolds (of arbitrary codimension) in Euclidean space in terms of mean curvature and volume.

Theorem 2.2. Given a Riemannian manifold $(M, g)$, constants $A_{0}>0$ and $H_{0} \geq$ 0 and an integer $m>0$, there is a constant $D=D\left(M, g, A_{0}, H_{0}, m\right)$, depending only on $A_{0}, H_{0}, m$ and $(M, g)$, so that any surface $F \subset M$ of genus $m$ with the norm of the mean curvature vector bounded above by $H_{0}$ and area at most $A_{0}$ has diameter at most $D$.

Proof. We proceed by contradiction. Suppose $F_{j} \subset M$ is a sequence of surfaces satisfying the hypothesis with $\operatorname{diam}\left(F_{j}\right) \rightarrow \infty$. By Theorem 2.1 , on passing to a subsequence (which we also denote by $F_{j}$ ) we can construct a family of diffeomorphisms $\varphi_{j}: F_{j} \rightarrow F$ to a fixed surface so that the associated maps $F \rightarrow M$ converge. Let $d_{j}: F \times F \rightarrow \mathbb{R}$ be the corresponding distance functions on $F$. Then $\operatorname{diam}\left(F_{j}\right)=\sup _{(p, q) \in F \times F} d_{j}(p, q) \rightarrow \infty$.

By Theorem 2.1, the functions $d_{j}: F \times F \rightarrow \mathbb{R}$ converge to a continuous function $d: F \times F \rightarrow \mathbb{R}$, which is bounded as $F \times F$ is compact. It follows that the functions $d_{j}$ are uniformly bounded above by the maximum of $d$, a contradiction.

For further applications, we would like to say that the pseudo-metric is not too degenerate. We show that, in fact, the limit is in an appropriate sense 2dimensional. 
Theorem 2.3. The limiting metric d has fractal dimension 2.

Outline of the proof of Theorem 2.1. We first show that, on passing to subsequences, the parametrising maps have a limit. We then show that the distance functions associated to the pullback metrics are equicontinuous, and hence, on passing to a subsequence, have a limiting pseudo-metric.

The construction of the limiting map is modelled on Gromov's compactness theorem for $J$-holomorphic curves. However, the ingredients in our situation, for instance the Schwarz lemma and the monotonicity lemma, need to be proved using different techniques than those for $J$-holomorphic curves. Furthermore, unlike the case of $J$-holomorphic curves (or minimal surfaces), the surfaces we consider do not satisfy an elliptic partial differential equation, and hence we do not have regularity results.

Consider henceforth a sequence of surfaces $F_{i}$ in the manifold $M$ satisfying the above bounds on the mean curvature, area and genus. The surfaces $F_{i}$ have Riemannian metrics obtained by restriction from $M$. We shall consider distances with respect to this metric. We shall frequently replace the given sequence by a subsequence, for which we continue to use the same notation.

The first elementary observation (see Section 3) is that the upper bound on mean curvature ensures a uniform upper bound on the sectional curvature of the surfaces.

The basic strategy of the proof is to puncture the surfaces about an $\epsilon$-net and, on the complement, to consider the complete hyperbolic metric in the conformal class of $\iota^{*} g$. Here $\epsilon>0$ is a sufficiently small constant depending only on the geometry of the ambient manifold $M$. A monotonicity lemma we prove in Section 4 shows that there is a uniform bound on the size of the $\epsilon$-net. Hence by passing to a subsequence we may assume that the topological type of the punctured surfaces is fixed.

The Bers-Mumford compactness theorem says that, on passing to a subsequence, the hyperbolic surfaces have a limit which is a complete hyperbolic surface. The limiting surfaces in general have additional cusps created by pinching curves. Our goal is to construct a corresponding limit of maps. This depends on relating the hyperbolic metric on the surfaces to the metric obtained from $M$.

Specifically, we show that away from the cusps the identity map from the surface with the hyperbolic metric to itself, with the metric restricted from $M$, is uniformly continuous. The first step (Section 5) is an argument that says that we have a form of uniform continuity at one scale. This uses an extremal length argument and the fact that an $\epsilon$-net has been deleted. We then need an appropriate Schwarz lemma to conclude uniform continuity at all stages.

The version of Schwarz lemma that we prove (in Section [6) and use is for discs with small diameter with an upper bound on the sectional curvature given. However, this cannot be applied directly, as it requires a lower bound on the injectivity radius at the origin (of the pullback metric). We apply the Schwarz lemma indirectly by constructing a lift of an appropriate disc under the exponential map. Such a lift is obtained (in Section 13) by a geometric argument, making crucial use of an upper bound on the perimeter of the disc.

Following Gromov's proof of the compactness theorem for $J$-holomorphic curves, the above ingredients allow us to construct a map on the punctured surface (see Section (7). Finally, further arguments using the extremal length, as well as a slightly more intricate one using in addition the monotonicity lemma, allow us to show that limits can also be obtained near the punctures in Section 8 . 
Finally, similar arguments allow us to, on passing to a further subsequence, construct a limiting pseudo-metric. It is easy to see that the limiting map is Lipschitz, completing the proof of Theorem 2.1.

A word on notation. We shall be considering various surfaces called $F_{i}, \Sigma_{i}, \Sigma$, $\bar{\Sigma}$ and $F$. Here we clarify what these mean (they will also be defined in appropriate places in the text).

The surfaces $F_{i}$ are the given surfaces of bounded mean curvature, taken with their pullback metrics. The inclusion map from the surface $F_{j}$ into $M$ will be denoted by $i_{j}$. We shall frequently pass to subsequences without changing our notation.

We shall construct a surface $F$, which is topologically of the same type as $F_{i}$, with a pseudo-metric that is as a limit of the pullback metrics. This will be the domain of the limiting map $i: F \rightarrow M$.

The surfaces $\Sigma_{i}$ are obtained from $F_{i}$ by deleting a finite set of points, with the metric on $\Sigma_{i}$ being the unique hyperbolic metric that is conformally equivalent to the pullback metric on $F_{i}$. The natural inclusion maps of $\Sigma_{j}$ into $F_{j}$ and $M$ will be denoted by $\iota_{j}$ and $\hat{\iota}_{j}$. We shall construct a hyperbolic surface $\Sigma$ as a limit of the surfaces $\Sigma_{i}$. Finally, we shall compactify $\Sigma$ and make certain identifications at infinity to obtain a surface $\bar{\Sigma}$.

\section{Contents}

1. Introduction 3977

2. Statement of results 3979

3. Preliminaries 3981

4. A monotonicity lemma and $\epsilon$-nets 3983

5. Hyperbolic structures 3985

6. A Schwarz lemma 3986

7. Limits of punctured surfaces $\quad 3987$

8. Filling punctures 3988

9. Existence of a limiting map 3992

10. A limiting metric 3992

11. Fractal dimension of the limit 3994

12. Appendix A: Bounds on curvature and conjugate radius 3999

13. Appendix B: Lifting discs under the exponential map 4001

\begin{tabular}{ll} 
Acknowledgements & 4004 \\
\hline
\end{tabular}

References 4004

\section{Preliminaries}

We assume throughout that all manifolds (and surfaces) we consider are orientable. Let $M$ be a closed, smooth, Riemannian $n$-manifold which we fix throughout. For a fixed real number $H_{0} \geq 0$, we consider embedded surfaces $F \subset M$ with mean curvature bounded above in absolute value by $H_{0}$. In the case $H_{0}=0$, these are just minimal surfaces. We further restrict ourselves to surfaces with area bounded above by $A_{0}$ and with a fixed genus $g$. The proofs of Lemma 3.1 and Lemma 3.2 are given in Appendix A. 
3.1. Upper bounds on curvature. We begin by observing that there is an upper bound on the sectional curvature of the surfaces. This is used for a version of the Schwarz lemma.

Lemma 3.1. There is a constant $K_{0}$ so that the sectional curvature of each surface $F_{i}$ is bounded above by $K_{0}$.

3.2. Lower bound on conjugate radius. Let $(F, g)$ be a Riemannian manifold. The conjugate radius at $p \in F$ is the largest $R$ such that $\exp _{p}$ is an immersion on $B(0, R) \subset T_{p}(M)$.

Lemma 3.2. Let $(F, g)$ be a complete Riemannian 2-manifold with sectional curvature bounded above by $K_{0}$. Then the conjugate radius at any $p \in F$ is at least $R=\frac{\pi}{3 \sqrt{K_{0}}}$. Moreover, if we write

$$
\exp ^{*}(g)=d r^{2}+f^{2}(r, \theta) d \theta^{2}
$$

for polar coordinates $(r, \theta)$ on $T_{p} F$ and $r<R$, then $f(r, \theta)$ increases as a function of $r$ and $f(r, \theta)>r / 2$ for all $\theta$.

3.3. Lifting discs under the exponential map. We need to use the geodesic coordinates of Lemma 3.2 for a topological disc $D \subset F$ in a surface with an upper bound on the sectional curvature. However, the injectivity radius may be less than the diameter of $D$. We shall see, however, that we can lift discs with small diameter and small boundary under the exponential map. Let $R=R\left(K_{0}\right)$ be the constant from Lemma 3.2

Lemma 3.3. Let $\iota: B \rightarrow(F, g)$ be an immersion of a disc into a complete Riemannian 2-manifold $(F, g)$ with sectional curvature bounded above by $K_{0}$. Suppose that for the pullback metric $i^{*} g$, the length of $\gamma=\partial B$ and the distance of a point in $B$ to $\gamma$ are both bounded above by $\epsilon<R / 10$, where $R=\frac{\pi}{3 \sqrt{K_{0}}}$. Then for $x=\iota(y)$ in the image of $B$, there is a lift $\tilde{\iota}$ of $\iota$ to the tangent space $T_{x} F$ so that $\iota=\exp _{x} \circ \tilde{\iota}$. Furthermore, the lift can be chosen so that $\tilde{\iota}(y)$ is the origin.

We remark that $\iota$ (at least restricted to the interior of the disc $B$ ) is often the inclusion map on a subset of $F$. Hence we identify $p$ with $x$.

We prove this in Section 13

3.4. Conformal moduli of annuli. We recall some basic results regarding the conformal moduli of annuli that will be used extensively. An annulus is a 2-manifold homeomorphic to the product of a circle and an interval. We consider annuli with a given conformal class of Riemannian metrics (i.e., a conformal structure). Recall that this is equivalent to specifying a complex structure.

A right circular annulus $A(H, W)$ is the Riemannian product of a circle of circumference $W$ and an open interval of (possibly infinite) length $H$. The following is the uniformisation theorem for annuli.

Theorem 3.4. We have the following:

(1) Any annulus $A$ with a conformal structure is conformally equivalent to a right circular annulus.

(2) $A\left(H_{1}, W_{1}\right)$ is conformally equivalent to $A\left(H_{2}, W_{2}\right)$ if and only if $H_{1} / W_{1}=$ $\mathrm{H}_{2} / W_{2}$. 
From the above result, it is immediate that the following definition gives a welldefined number in $(0, \infty]$.

Definition 3.5. The modulus $\operatorname{Mod}(A)$ of an annulus $A$ with a conformal structure is $\operatorname{Mod}(A)=H / W$, where $A$ is conformally equivalent to the right circular annulus $A(H, W)$.

By definition the modulus is a conformal invariant. Further, two annuli are conformally equivalent if and only if they have the same modulus.

Observe that the area of a right circular annulus $A=A(H, W)$ is $H W$, so the modulus of $A(H, W)$ can also be expressed as $\operatorname{Mod}(A)=\operatorname{Area}(A) / W^{2}$, i.e., $W^{2}=\operatorname{Area}(A) / \operatorname{Mod}(A)$. The following fundamental (though elementary) result of Ahlfors allows one to get an upper bound on the appropriate width for an annulus.

Theorem 3.6 (Ahlfors). Let $A$ be an annulus with a conformal structure. Then there is a simple closed curve $\gamma \subset A$ separating the two boundary components of $A$ whose length $l(\gamma)$ satisfies

$$
l(\gamma)^{2} \leq \operatorname{Area}(A) / \operatorname{Mod}(A) .
$$

Furthermore, given an identification of the annulus with $S^{1} \times J$ for an interval $J$, we can find a curve $\gamma$ as above of the form $S^{1} \times\{p\}$.

It is easy to see that an annulus obtained from a disc by puncturing a point has infinite modulus. Further, if we take an open disc $D(r)$ of a fixed radius $r$ in Euclidean or hyperbolic space and $D(\rho)$ is the concentric disc of radius $\rho<r$, then the modulus of the annulus $A=D(r)-\overline{D(\rho)}$ goes to infinity as $\rho \rightarrow 0$.

3.5. Real-analytic metrics and the cut-locus. Let $(F, g)$ be a Riemannian manifold and $p \in F$. Let

$$
U_{p}:=\left\{v \in T_{p} M \mid \exp _{p}(t v) \text { is a minimal geodesic on }[0,1]\right\} .
$$

Note that the boundary $\partial U_{p}$ is the cut-locus in $T_{p} M$.

In case $(F, g)$ is real-analytic we have the following proposition which follows from results of S. B. Myers.

Theorem 3.7 (S. B. Myers [9, [10])). Let $(F, g)$ be a closed real-analytic Riemannian 2-manifold and $p \in F$. Then:

(i) $\partial U_{p}$ is a piecewise-smooth 1-manifold homeomorphic to $S^{1}$.

(ii) Suppose that the sectional curvature of $(F, g)$ is bounded above by $K_{0}$. Let $R=\frac{\pi}{3 \sqrt{K_{0}}}$ be the lower bound on conjugate radius given by Lemma 3.2, If $\delta<R$, then the geodesic sphere $\partial B(p, \delta)$ is a disjoint union of piecewise-smooth circles.

\section{A monotonicity LEMma AND $\epsilon$-NETS}

As in the case of the monotonicity lemma for minimal surfaces, the proof of our monotonicity lemma is based on an isoperimetric inequality. The relevant isoperimetric inequality is due to Hoffman and Spruck. This will also be used later on in the paper.

Theorem 4.1 (Hoffman-Spruck [6]). Let $\Sigma$ be a compact surface with boundary in a Riemannian n-manifold $(M, g)$. There is a constant $v_{0}=v_{0}(M, g)$ such that 
either $\operatorname{vol}(\Sigma) \geq v_{0}$ or

$$
\operatorname{vol}(\Sigma)^{\frac{1}{2}} \leq \beta\left(\operatorname{vol}(\partial \Sigma)+\int_{\Sigma}|H| d V_{\Sigma}\right)
$$

where $\beta$ is an absolute constant.

Proof. This is a corollary of Theorem 2.2 of [6]. In the notation of that paper take $\alpha=\frac{1}{2}$ and $b=1$, for instance. Let $i_{0}$ denote the injectivity radius of $M$. Then either (11) holds or

$$
\operatorname{vol}(\Sigma) \geq v_{0}=\frac{\omega_{2}}{2} \min \left\{1, \frac{i_{0}^{2}}{\pi^{2}}\right\}
$$

The main result of this section is a monotonicity lemma, giving a lower bound on the area of small balls.

Theorem 4.2. Let $(M, g)$ be a Riemannian n-manifold and $\Sigma$ a compact surface in $M$ with mean curvature $|H| \leq H_{0}$. There exist $c=c\left(M, g, H_{0}\right)$ and $\delta=\delta\left(M, g, H_{0}\right)$ such that the volume of any ball of radius $\epsilon \leq \delta$ in $\Sigma$ with the induced metric satisfies

$$
\operatorname{vol}(B(p, \epsilon)) \geq c \epsilon^{2} .
$$

Proof. By making an arbitrarily small $C^{2}$-perturbation we can assume that $g$ is real-analytic. By Theorem 3.7 this implies that the boundary of a ball of radius $\epsilon$ less than $R$ is piecewise-smooth. We apply Theorem 4.1 to the manifold with piecewise-smooth boundary $\partial B(p, \epsilon)$. Even (11) is stated for submanifolds with smooth boundaries. It is clearly true even if the boundaries are piecewise-smooth, as can be seen by exhausting such manifolds by submanifolds with smooth boundaries. We will apply it to the metric balls $B(p, r), 0<r \leq \epsilon$.

If $\operatorname{vol}(B(p, r)) \geq v_{0}$ for some $r<\epsilon$, then $\operatorname{vol}\left(B(p, \epsilon)>\operatorname{vol}(B(p, r)) \geq v_{0}\right.$. Hence $\operatorname{vol}(B(p, \epsilon)) \geq \epsilon^{2}$ if $\epsilon<\sqrt{v_{0}}$.

So we can suppose that the isoperimetric inequality (1) holds for every $r \leq \epsilon$.

It follows from the co-area formula that $\operatorname{vol}(B(p, r))=\int_{0}^{r} \operatorname{vol}(\partial B(p, t)) d t$. Hence $\operatorname{vol}(B(p, r))$ is differentiable a.e. as a function of $r$.

By Theorem 4.1, we then have

$$
\begin{aligned}
\frac{d}{d r} \operatorname{vol}(B(p, r)) & =\operatorname{vol}(\partial B(p, r)) \\
& \geq \beta^{-1} \operatorname{vol}(B(p, r))^{\frac{1}{2}}-\int_{B(p, r)}|H| d V_{\Sigma} \\
& \geq \beta^{-1} \operatorname{vol}(B(p, r))^{\frac{1}{2}}-H_{0} \operatorname{vol}(B(p, r)) \quad \text { a.e. }
\end{aligned}
$$

We next see that we can assume $\beta^{-1} \operatorname{vol}(B(p, r))^{\frac{1}{2}}>2 H_{0} \operatorname{vol}(B(p, r))$ for $0 \leq$ $r \leq \epsilon$. If not, then we get $\operatorname{vol}(B(p, \epsilon)) \geq \operatorname{vol}\left(B(p, r) \geq\left(\frac{1}{2 \beta H_{0}}\right)^{2}\right.$, which is larger than $\epsilon^{2}$ for $\epsilon<2 \beta H_{0}$.

As $\beta^{-1} \operatorname{vol}(B(p, r))^{\frac{1}{2}}>2 H_{0} \operatorname{vol}(B(p, r))$, we get

$$
\frac{d}{d r} \operatorname{vol}(B(p, r)) \geq \frac{1}{2 \beta} \operatorname{vol}(B(p, r))^{\frac{1}{2}} .
$$

By integrating, we get $\operatorname{vol}\left(B(p, \epsilon)>\beta^{\prime} \epsilon^{2}\right.$ with $\beta^{\prime}=\frac{1}{16 \beta^{2}}$. 
Hence we can take

$$
\delta=\min \left\{R, \sqrt{v_{0}}, 2 \beta H_{0}\right\}, \quad c=\min \left\{1, \frac{1}{16 \beta^{2}}\right\} .
$$

Gromov's proof of the compactness of $J$-holomorphic curves is based on puncturing along an $\epsilon$-net. We shall choose an appropriate constant $\epsilon=\epsilon\left(M, H_{0}, A_{0}, g_{0}\right)$, which is the same for all the surfaces $S_{i}$. Assume that such a constant has been chosen. For each surface $F_{i}$, we choose a maximal subset $S_{i} \subset F_{i}$ so that the distance between every pair of points in $S_{i}$ is at least $\epsilon$, i.e., $S_{i}$ is an $\epsilon$-net.

Lemma 4.3. There is a constant $N$ such that for all $i,\left|S_{i}\right| \leq N$.

Proof. Fix a surface $F_{i}$ in the sequence. By hypothesis, the open balls $B(x, \epsilon) \subset F_{i}$, $x \in S_{i}$, are disjoint. By the monotonicity lemma, there is a constant $a$ such that each of the balls have area at least $a$. As the area of $F_{i}$ is bounded above by $A_{0}$, the cardinality of $S_{i}$ is bounded above by $A_{0} / a$.

\section{Hyperbolic StruCtures}

Consider the sequence of surfaces $\hat{F}_{j}=F_{j}-S_{j}$. As the cardinality of $S_{j}$ and the genus of $F_{j}$ are bounded above, by passing to a subsequence we can, and do, assume that the surfaces $\hat{F}_{j}$ are of a fixed topological type. Further, by ensuring that the number of punctures is at least three, we can ensure that $\chi\left(\hat{F}_{i}\right)<0$. By the uniformisation theorem, there is a unique complete hyperbolic metric on $\hat{F}_{j}$ that is conformal to the given Riemannian metric. We view this as a hyperbolic surface $\Sigma_{j}$ which is identified with a subset of $F_{j}$.

By the Bers-Mumford compactness theorem, on passing to a subsequence the surfaces $\Sigma_{j}$ converge to a complete, finite volume, hyperbolic surface $\Sigma$. More concretely, we have a sequence of numbers $\delta_{j} \rightarrow 0$, compact sets $\kappa_{j} \subset \Sigma_{j}$ and $\left(1+\delta_{j}\right)$-bi-Lipschitz diffeomorphisms $\psi_{j}: \kappa_{j} \rightarrow \Theta_{j} \subset \Sigma$ so that the sets $\Theta_{j}$ form an exhaustion of $\Sigma$. Furthermore, by passing to smaller sets, we can ensure that the sets $\Theta_{j}$ are complements of horocyclic neighbourhoods of the cusps of $\Sigma_{j}$ (with the intersection of the neighbourhoods of each cusp being empty).

We shall show that the maps $\iota_{j} \circ \psi_{j}^{-1}$ are equicontinuous on compact sets (in a sense made precise below) so that the Arzela-Ascoli theorem allows us to construct a limiting map $\iota: \Sigma \rightarrow M$. We shall henceforth implicitly identify subsets of $\Sigma$ (contained in $\Theta_{j}$ ) with subsets of $\kappa_{j}$ using the maps $\psi_{j}$. Under these identifications, the maps $\iota_{j}$ can be regarded as maps on subsets of $\Sigma$.

Now consider a compact set $K \subset \Sigma$. The injectivity radius on $K$ is bounded below by a constant $\alpha>0$. For $j$ large, as above we can identify $K$ with subsets $K_{j} \subset \Sigma_{j}$, and the injectivity radius on these sets is also bounded below by $\alpha$, as the map $\psi_{j}$ is $\left(1+\delta_{j}\right)$-bi-Lipschitz with $\delta_{j}$ small for $j$ large.

Our first step in proving equicontinuity is an upper bound on the diameter in the pullback metric of small hyperbolic balls of a fixed size.

Lemma 5.1. There is a constant $r=r\left(\epsilon, \alpha, A_{0}, H_{0}, m, M\right)$ such that for any point $x \in K_{j}, \iota_{j}(B(x, r))$ is contained in a smooth (not in general metric) ball $B(\gamma)$ in $\widehat{F_{j}} \subset F_{j}$ of diameter $3 \epsilon$ whose boundary has length at most $\epsilon$. 
Proof. We shall choose $r<\alpha$ appropriately. For a point $x \in K_{j}$, consider the annulus $A=B(x, \alpha) \backslash \operatorname{int}(B(x, r))$. Choose $r$ small enough that this annulus has modulus at least $A_{0} / \epsilon^{2}$. Note that this depends only on $\alpha, A_{0}$ and $\epsilon$.

Consider the annulus $\iota_{j}(A) \subset F_{j}$. This has area bounded above by $A_{0}$. By Theorem 3.6, there is a curve $\gamma$ in $\iota_{j}(A)$, separating the boundary components of $A$, so that the length of $\gamma$ is bounded above by $\epsilon$. The curve $\gamma$ is the boundary of a ball $B(\gamma)$ that contains $\iota_{j}(B(x, r))$. We shall show that $B(\gamma)$ has diameter at most $3 \epsilon$.

As $B(\gamma)$ is a ball whose boundary is a connected set of diameter at most $\epsilon$, it suffices to show that for each point $x \in B(\gamma)$, the distance of $x$ from the boundary is at most $\epsilon$. To see this, observe that $B(\gamma) \subset F_{j}-S_{j}$ by construction. As $S_{j}$ is an $\epsilon$-net, the distance from $x \in B(\gamma)$ to some point $y \in S_{j}$ is at most $\epsilon$. As the metric on $F_{j}$ is obtained from a Riemannian metric and $F_{j}$ is compact, there is a path $\beta$ from $x$ to $y$ of length at most $\epsilon$. This path must intersect the boundary of $B(\gamma)$ at some point $z$. It follows that $d(x, z)<\epsilon$.

\section{A Schwarz Lemma}

To deduce equicontinuity of the maps $\iota_{j}: \Sigma_{j} \rightarrow \hat{F}_{j}$ from the estimate on diameters in the induced metrics of balls of radius $\rho$, we use an appropriate Schwarz lemma.

Theorem 6.1. Let $(S, g)$ be a Riemannian 2-manifold and $p \in \Sigma$ such that the sectional curvature of $S \leq K_{0}$ and the injectivity radius $\operatorname{inj}(p) \geq i_{0}$. Let $\left(\mathbb{B}^{2}, h\right)$ denote the unit disc with the Poincaré metric of curvature -1 .

For any $r>0$, there exists $\eta=\eta\left(K_{0}, i_{0}\right)$ such that for any conformal map

$$
f: B_{h}(0, r) \subset \mathbb{B}^{2} \rightarrow B_{g}(p, \eta) \subset S,
$$

with $f(0)=p$, we have

$$
\left\|d f_{0}(v)\right\|_{g} \leq r^{-2}\|v\|_{h}
$$

for all $v \in T_{0} \mathbb{B}^{2}$.

Proof. The idea is to conformally deform $g$ on $B_{g}(p, \eta)$ to $\tilde{g}=\exp (2 u) g$ so that $\tilde{g}$ has curvature $\leq-1$. The deformation will be done so that we have control over the conformal factor. Then we can apply the Ahlfors-Schwarz Lemma in the new metric to get the required estimate.

First choose $\eta<\operatorname{inj}(p)$. Define $u: B_{g}(p, \delta) \rightarrow \mathbb{R}^{+}$by

$$
u(x)=\lambda^{2} \rho^{2}(x) \text { and } \lambda=\sqrt{\frac{K_{0}}{2}+1},
$$

where $\rho(x)$ is the distance of $x$ from $p$. Since $\delta<\operatorname{inj}(p), u$ is smooth. Now the curvature $\tilde{K}$ of $\tilde{g}=\exp (2 u) g$ is related to the curvature $K$ of $g$ by

$$
\tilde{K}=(K-\triangle u) \exp (-2 u),
$$

where $\triangle$ is the negative Laplacian.

Since $K \leq K_{0}$, if $\rho(x)<\min \left\{\operatorname{inj}(p), \frac{\pi}{4 \sqrt{K_{0}}}\right\}$, we can apply the usual comparison theorem for the Laplacian of a distance function. Here the comparison space $S_{0}$ is the sphere of curvature $K_{0}$. Let $p_{0}, x_{0}$ denote points in $S_{0}$ corresponding to $p, x$ 
and let $\rho_{0}$ denote the distance from $p_{0}$. We have $\rho_{0}\left(x_{0}\right)=\rho(x)$. The comparison theorem gives

$$
(\triangle \rho)(x) \geq\left(\triangle_{0} \rho_{0}\right)\left(x_{0}\right)=\cot \left(\sqrt{K_{0}} \rho(x)\right)=\frac{\cos \left(\sqrt{K_{0}} \rho(x)\right)}{\sin \left(\sqrt{K_{0}} \rho(x)\right)} .
$$

In particular,

$$
(\triangle \rho)(x) \geq 0,
$$

if $\rho(x)<\min \left\{\operatorname{inj}(p), \frac{\pi}{4 \sqrt{K_{0}}}\right\}$. For such $x$, we have

$$
\begin{aligned}
(\triangle u)(x) & =\lambda^{2}\left(\triangle \rho^{2}\right)(x) \\
& =2 \lambda^{2} \rho(x)(\triangle \rho)(x)+2 \lambda^{2}\|d \rho\|^{2}(x) \\
& \geq 2 \lambda^{2} .
\end{aligned}
$$

We then have

$$
\tilde{K}(x) \leq\left(K_{0}-2 \lambda^{2}\right) \exp \left(-2 \rho(x)^{2}\right)=-2 \exp \left(-2 \rho(x)^{2}\right) \leq-1,
$$

for $\rho(x)<\sqrt{\frac{\log (2)}{2}}$. Hence we can take

$$
\eta=\frac{1}{2} \min \left\{i_{0}, \frac{\pi}{4 \sqrt{K_{0}}}, \frac{\log (2)}{2}\right\} .
$$

Now we modify the metric on $B_{h}(0, r)$. Note that if $\phi(z)=r^{2} \frac{\left(1-|z|^{2}\right)^{2}}{\left(r^{2}-|z|^{2}\right)^{2}}$, then $\left(B_{h}(0, r), \phi^{2} h\right)$ is a complete Riemannian manifold of constant curvature -1 .

Let us recall the

Ahlfors-Schwarz Lemma: Let $\left(F_{1}, g_{1}\right)$ be a complete Riemannian 2-manifold with curvature $\geq-c$ and $\left(F_{2}, g_{2}\right)$ a Riemannian 2-manifold with curvature $\leq-d$, where $c, d>0$. If $f:\left(F_{1}, g_{1}\right) \rightarrow\left(F_{2}, g_{2}\right)$ is a conformal map, then

$$
\left\|d f_{x}(v)\right\|_{g_{2}} \leq \sqrt{\frac{c}{d}}\|v\|_{g_{1}}
$$

for all $x \in F_{1}, v \in T_{x} F_{1}$.

We apply the Ahlfors-Schwarz Lemma to $f:\left(B_{h}(0, r), \phi^{2} h\right) \rightarrow\left(B_{g}(p, \eta), \exp (2 u) g\right)$ at $x=0$. Noting that $c=d=1, \phi(0)=r^{-2}$ and $\exp (2 u(p))=1$, we get

$$
\left\|d f_{0}(v)\right\|_{g} \leq r^{-2}\|v\|_{h} .
$$

\section{Limits OF PUNCTURED SURFACES}

The Schwarz lemma of Theorem 6.1 requires a lower bound on the injectivity of the pullback metric, which we cannot control. However, the following consequence of Lemma 3.3 allows us to obtain uniform Lipschitz bounds.

Note that the surface $\Sigma$ can be identified with the complement of a collection of annuli in $\Sigma_{j}$ for each $j$. Furthermore, if $K \subset \Sigma$ is a compact set, then for $j$ large the corresponding set $K_{j} \subset \Sigma_{j}$ is close to an isometry.

Let $K \subset \Sigma$ be a compact set. Then there is a constant $\alpha>0$ so that for $j$ large, the injectivity radius of the set $K_{j} \subset \Sigma_{j}$ corresponding to $K$ is bounded below by $\alpha$. Let $\iota_{j}: K_{j} \rightarrow F_{j}$ be the inclusion map. 
Lemma 7.1. There is a constant $\kappa$, independent of $j$, so that the map $\iota_{j}$ is $\kappa$ Lipschitz for $j$ sufficiently large.

Proof. By Lemma 5.1 there is a constant $r>0$ such that for any $p \in K_{j}, \iota_{j}(B(p, r))$ is contained in a topological ball $B(\gamma)$ whose diameter is at most $3 \epsilon$ and whose boundary has length at most $\epsilon$ in the pullback metric. Here $B(p, r)$ is the ball in the hyperbolic metric on $\Sigma_{j}$.

By Lemma 3.3, there is a lift $\tilde{\iota}_{j}$ of $\iota_{j}$ to $T_{p} F_{j}$ under the exponential map $\exp _{p}$. Choose an isometry $\phi: B_{h}(0, r) \rightarrow B(p, r)$, with $\phi(0)=p$, where $B_{h}(0, r)$ denotes a ball in the Poincaré disc as in Theorem 6.1 .

We can now apply the Schwarz lemma of Theorem 6.1 to

$$
f=\tilde{\iota}_{j} \circ \phi: B_{h}(0, r) \rightarrow B(0, R) \subset T_{p} F,
$$

where $B(0, R)$ is endowed with the metric $\exp _{p}^{*} g$.

Since $\iota_{j}=\exp _{p} \circ \tilde{\iota}$ and $\exp _{p}:\left(B(0, R), \exp _{p}^{*} g\right) \rightarrow\left(F_{j}, g\right)$ is an isometry, we have

$$
\left\|d \iota_{j}\right\|_{\phi(x)}=\left\|d \tilde{\iota}_{j}\right\|_{\phi(x)}=\|d f\|_{x} .
$$

Hence we obtain an upper bound on $\left\|d \iota_{j}\right\|$ at $p$ for the map $\iota_{j}: K_{j} \rightarrow \hat{F}_{j} \hookrightarrow M$. Note that this upper bound does not depend on $j$, but depends only on $M$ and $\alpha$. This gives the uniform Lipchitz bound.

Lemma 7.2. On passing to a subsequence, the maps $\hat{\iota}_{j}=i_{j} \circ \iota_{j}: \Sigma_{j} \rightarrow \hat{F}_{j} \rightarrow M$ converge uniformly on compact sets to a map $\iota: \Sigma \rightarrow M$.

Proof. Let $K \subset \Sigma$ be a compact set. We apply Lemma 7.1 to the restrictions of the maps $\iota_{j}$, regarded as maps on $K$, to obtain a uniform Lipshitz bound. Thus, by the Arzela-Ascoli theorem, there is a subsequence of surfaces so that the maps $\iota_{j}$ converge to a map $\iota: K \rightarrow M$. Now consider an exhaustion of $\Sigma$ by compact sets $K^{(i)}$. By the above, we can find a subsequence of surfaces to obtain a limit on $K^{(1)}$. On passing to a further subsequence, we obtain a limit on $K^{(2)}$. Iterating this process and using a diagonal subsequence as usual, we obtain a limiting map on $\Sigma$.

\section{FilLing PUNCTURES}

We have constructed a limiting map from the punctured surface $\Sigma$ of finite type to the manifold $M$. We now show that, on passing to a further sequence, we can construct a limiting map on a closed surface $\bar{\Sigma}$ obtained from $\Sigma$. This is the surface constructed by filling in the punctures and making certain identifications of the filled-in points.

Observe that there are two kinds of punctures (cusps). The first kind are those that correspond to the limits of punctures in $\Sigma_{j}=\hat{F}_{j}$ corresponding to points of the $\epsilon$-net $S_{j}$. We denote the set of such punctures as $S(\Sigma)$. We can, and shall, identify these with points on the surfaces $F_{j}$.

The second kind are pairs of punctures formed in passing to the compactification of Moduli space by a sequence of curves $\alpha_{j} \subset \Sigma_{j} \subset F_{j}$ whose length in the hyperbolic metric on $\Sigma_{j}$ goes to zero. We denote the set of such pairs of cusps by $\Lambda(\Sigma)$. Each point $p \in \Lambda(\Sigma)$ corresponds to a pair of ends $p^{ \pm}$of $\Sigma$.

We consider the Freudenthal (end-point) compactification of $\Sigma$ (where one point is added for each puncture) and identify points corresponding to pairs of punctures $p^{ \pm}, p \in \Lambda(\Sigma)$. We denote the resulting surface by $\bar{\Sigma}$. 
Thus,

$$
\bar{\Sigma}=\Sigma \coprod \mathcal{S}(\Sigma) \coprod \Lambda(\Sigma) .
$$

We shall extend the inclusion map to $\bar{\Sigma}$. As there are only finitely many punctures, it suffices to show that we can extend the map to the point in $\bar{\Sigma}$ corresponding to each puncture or pair of punctures.

We first consider a point $z \in S(\Sigma)$ corresponding to a limit of points of $z_{j} \in S_{j}$. As $M$ is compact, by passing to a subsequence we can ensure that $\iota_{j}\left(z_{j}\right)$ converges to a point, which we take to be the image of $z$ in the limiting map. It remains to show that this extension is continuous.

Suppose $D(z)$ is a closed disc in $\bar{\Sigma}$ containing the point $z$ in its interior and no other points of $S(\Sigma)$ and $\Lambda(\Sigma)$. Then for $j$ sufficiently large, the disc $D(z)$ can be identified with discs $D_{j}=D_{j}(z) \subset F_{j}=\Sigma_{j}$. Continuity is immediate from the following lemma.

Lemma 8.1. Given $\delta>0$, there is a disc $D(z)$ as above such that for $j$ sufficiently large, the diameter of $\iota_{j}\left(D_{j}\right)$ is at most $\delta$.

We first give a brief sketch of the proof. Using an appropriate extremal length argument, we enclose $D_{j}$ in a disc whose boundary has a small perimeter. As before, we lift this disc under the exponential map. We then use polar co-ordinates, for which we have obtained the appropriate estimates in Section 3.2. These allow us to deduce a bound on the diameter of the disc from the bound on the perimeter. We now turn to the details.

Proof of Lemma 8.1. As the modulus of a disc punctured at a point is infinite, we can choose $D(z)$ so that there is an annulus $A(z)$ enclosing the puncture corresponding to $z$ so that the modulus of the annulus is at least $M$, where $M$ is any specified number. The annulus $A(z)$ corresponds to an annulus $A$ in $F_{j}$ which, for $j$ sufficiently large, has modulus greater than $M$. Fix such a $j$.

Choosing $M$ sufficiently large and using an extremal length argument as in Lemma 5.1, we can find a curve $\gamma$ in $A$ enclosing $z_{j}$ with length $L(\gamma)$ less than $\eta$ in the pullback metric, with $\eta>0$ to be specified. Let $B(\gamma)$ be the disc bounded by $\gamma$ enclosing $z_{j}$. We shall show that if $\eta$ is small enough (depending only on $\delta$ and $M)$, then the disc $D_{j}$ has diameter less than $\delta$. This implies that the diameter of the image $\iota_{j}\left(F_{j}\right)$ is less than $\delta$.

We choose $\eta<\min (\delta / 2, \epsilon / 2)$. As the length of the boundary $\gamma$ of $B(\gamma)$ is bounded by $\delta / 2$, it suffices to show that the distance from a point $x_{0}$ of $B(\gamma)$ to $\gamma$ is at most $\delta / 3$. Suppose this is not the case; find a point $x_{0}$ with distance from the boundary greater than $\delta / 3$.

Consider the exponential map from the tangent space at $x_{0}$. We have seen that this is an immersion on the ball of radius 10€. Further, as before the diameter of the set $D_{j}$ is bounded by $2 \epsilon$. We choose a lift as in Lemma 3.3 so that the image of $x_{0}$ is the origin.

We now recall Lemma 3.2 .

We have assumed that the distance between $x_{0}$ and $\gamma$ is greater than $\delta / 3$. Hence the lift of $\gamma$ (which we continue to denote by $\gamma$ ) encloses the ball of radius $\delta$ around the origin.

Consider the radial projection $p: \gamma \rightarrow \alpha$ of $\gamma$ onto the boundary $\alpha$ of the ball of radius $\delta / 3$. As $f(r, \theta)$ is an increasing function of $r$, this is distance decreasing. 
Thus, if $d s_{\gamma}$ and $d s_{\alpha}$, denote the oriented arc lengths of the respective curves, $p^{*}\left(d s_{\alpha}\right)=\psi \cdot d s_{\gamma}$, with $\psi \leq 1$.

As $\gamma$ encloses the origin, the projection has degree one (after possibly reversing the orientation of $\gamma$ ). Thus, we have

$$
l(\alpha)=\int_{\alpha} d s_{\alpha}=\int_{\gamma} p^{*}\left(d s_{\alpha}\right)=\int_{\gamma} \psi \cdot d s_{\gamma} \leq \int_{\gamma} d s_{\gamma}=l(\gamma) .
$$

Hence $l(\alpha) \leq l(\gamma)<\eta$. Now by Lemma 3.2, it follows that $l(\alpha)>\pi \delta / 3$. Hence, as $\eta<\delta / 2<\pi \delta / 3$ we get a contradiction.

We now turn to the case of a point $p \in \Lambda(\Sigma)$, which corresponds to a pair of ends $p^{ \pm}$. As before, we can find disc neighbourhoods of these, $D^{ \pm}(p)$, enclosed by annuli $A^{ \pm}(p)$ of modulus at least $\mu$ (see Figure 1), with $\mu$ to be specified. We denote the boundaries of the disc by $\xi^{ \pm}=\partial D^{ \pm}$.

Now consider a surface $\Sigma_{j}$ with $j$ large. The curves $\xi_{j}^{ \pm}$in $F_{j}$ corresponding to $\xi^{ \pm}$enclose an annulus $B_{j}$ of large modulus (for $j$ large) and hence are separated by a curve $\alpha_{j}=\alpha_{j}(p)$ of length $l_{j}$ (in the pullback metric on $F_{j}$ ) so that as $j \rightarrow \infty$, $l_{j} \rightarrow 0$. Hence we can pass to a subsequence so that the images $\iota_{j}\left(\alpha_{j}\right)$ converge to a point $y$ in $M$. We extend the map so that the point $p$ maps to $y$. We show that this extension is continuous.

Lemma 8.2. Given $\delta>0$, there are discs $D^{ \pm}=D^{ \pm}(p)$ as above so that for $j$ sufficiently large the diameter of $B_{j}=B_{j}(p)$ is at most $\delta$.

Note that in this case the upper bounds on the curvature and the length of the boundary do not suffice, as there are flat annuli with arbitrarily short boundaries with arbitrarily large diameters. We shall use an indirect argument, which we sketch below.

Consider an annulus as above whose boundary curves have length less than a small real number $\delta$. As the annulus is contained in the complement of an $\epsilon$-net, we can bound the distance between the two boundary components. This, together with the bound on the boundary components and the upper bound on curvature, gives an upper bound on the area of the annulus. By ensuring that $\epsilon$ (hence $\delta$ ) is sufficiently small, we can thus ensure that we are not in the bubbling case of the isoperimetric inequality (i.e., we ensure that volume is less than $v_{0}=v_{0}(g)$ of Theorem 4.1). Hence, as the upper bound $\delta$ on the perimeter tends to zero, so does the area. Using the monotonicity lemma, we can deduce that the diameter also tends to 0 . We now turn to the details.

Proof of Lemma 8.2. As the annuli $A_{j}^{ \pm}$have modulus greater than $\mu$, by choosing $\mu$ sufficiently large we can ensure that there are curves $\gamma_{j}^{ \pm}$separating the boundary components of the annuli with lengths at most $\epsilon$. We let $C_{j}=C_{j}(p)$ be the annulus enclosed by the curves $\gamma_{j}^{ \pm}$. We shall show that the diameter of $C_{j}$ is small, from which a bound on the diameter of the image of $B_{j}$ follows.

Observe that as the annulus $C_{j}$ is contained in the complement of an $\epsilon$-net in $F_{j}$, as before the boundary components are at most a distance $2 \epsilon$ apart. Namely, as before, each point is a distance less than $\epsilon$ from at least one of the boundary components. By connectedness, some point must have distance less than $\epsilon$ from both of the boundary components.

Choose an embedded arc $\beta$ of length at most $2 \epsilon$ joining the boundary components. We obtain a disc $\Delta_{j}$ from the annulus $C_{j}$ by splitting along the curve $\beta$. 

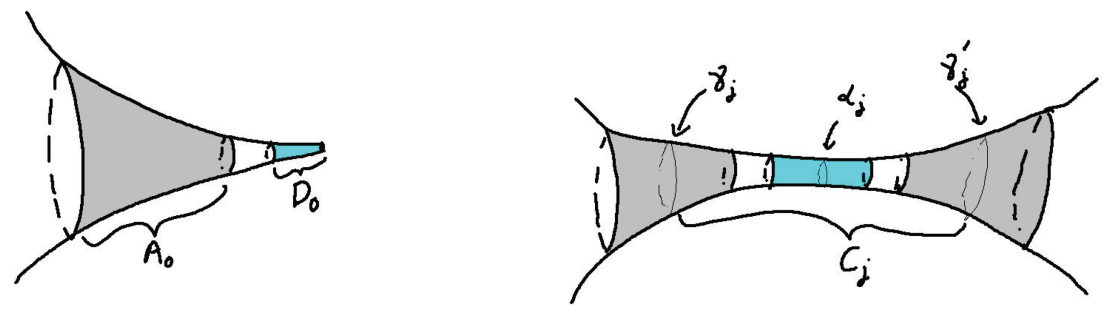

FiguRE 1. Filling a cusp from the compactification

Observe that all the above results continue to hold when the inclusion map of the disc is replaced by the quotient map from the disc to $A_{j}$. Choosing $\eta<\epsilon / 2$, the disc $\Delta_{j}$ has boundary of length at most $6 \epsilon$.

As before, we pick a point $x_{0}$ in $B$ and find a lift of $\Delta_{j}$ with respect to the exponential map based at $x_{0}$ so that the lift of $x_{0}$ is the origin. Recall that there is a constant $v_{0}=v_{0}(B(g))>0$ associated with the isoperimetric inequality (Theorem 4.1) which depends only on $M$.

Lemma 8.3. If $\epsilon>0$ is chosen sufficiently small, depending only on $M$, then the area of $\Delta_{j}$ with the pullback metric is less than $v_{0}$.

Proof. As the origin is in the interior of $\Delta_{j}$ and the distance of any point in $B(\gamma)$ to the boundary is at most $\epsilon$, the disc $\Delta_{j}$ is contained in the ball of radius $\epsilon$ around the origin.

Using polar co-ordinates as before, the area form on $\Delta_{j}$ is $\omega=f(r, \theta) d r \wedge d \theta$. Let $F(r, \theta)=\int_{0}^{r} f(\rho, \theta) d \rho$ and $\alpha=F(r, \theta) d \theta$. Then the area form $\omega=d \alpha$; hence by Stokes's theorem

$$
\text { Area }=\int_{\Delta_{j}} \omega=\int_{\partial \Delta_{j}} \alpha
$$

Let $\zeta=\partial \Delta_{j}$. In polar co-ordinates, we can express $\zeta=\left(\zeta_{r}, \zeta_{\theta}\right)$. In terms of these co-ordinates,

$$
\text { Area }=\int_{\partial \Delta_{j}} \alpha=\int F(r, \theta) \frac{d \zeta_{\theta}}{d t} d t
$$

Note that as $f(r, \theta)$ is increasing as a function of $R, F(r, \theta) \leq \epsilon f(r, \theta)$ for $r<\epsilon$. Further, by Pythagoras's theorem the oriented arc length $d s$ satisfies $d s>\frac{d \zeta_{\theta}}{d t}$. Thus, it follows that

$$
\text { Area }=\int F(r, \theta) \frac{d \zeta_{\theta}}{d t} d t<\epsilon l(\zeta)
$$

As $l(\zeta)<5 \epsilon$, the lemma follows.

Assume $\epsilon>0$ has been chosen to satisfy the previous lemma. Then, by the isoperimetric inequality, if $\eta$ is sufficiently small, the volume of $C_{j}$ is less than $V(\eta)$, where $V(\eta)$ is a function of $\eta$ such that $V(\eta) \rightarrow 0$ as $\eta \rightarrow 0$.

We show that if $\eta>0$ is sufficiently small, then the diameter of the annulus $C_{j}$ is less than $\delta$. As before, it suffices to show that the distance of each point $x_{0} \in C_{j}$ to the boundary of the annulus is at most $\delta / 4$. 
Suppose the distance of $x_{0}$ to $\partial C_{j}$ is greater than $\delta / 4$. It follows by the monotonicity lemma that the area of $C_{j}$ is at least $V_{\delta}$, with $V_{\delta}$ depending only on $\delta$ and $M$. Choose $\eta$ such that $V(\eta)<V(\delta)$, to get a contradiction. Thus, we get a bound on the diameter showing continuity, as required. This completes the proof of Lemma 8.2

\section{EXISTENCE OF A LIMITING MAP}

We can now show that we have a limiting map from $F$. We have already constructed a limiting map on $\bar{\Sigma}$. We shall express $\bar{\Sigma}$ as the quotient of a surface $F$ and construct appropriate diffeomorphisms $\varphi: F_{j} \rightarrow F$ so that the maps $i_{j} \circ \varphi^{-1}$ converge.

Assume that we have chosen a subsequence so that we have a corresponding limit map $\iota$ on $\bar{\Sigma}$. Let $F$ be a surface of genus $m$. Identify $S(\Sigma)$ with a subset $S(F)$ of $F$. Then for a collection of disjoint curves $\alpha(p) \subset F-S(F), p \in \Lambda(\Sigma)$, we have a homeomorphism of $\Sigma$ with $F-\bigcup_{p \in \Lambda(\Sigma)} \alpha(p)-S(F)$. This extends continuously to a homeomorphism of the quotient of $F$ with each curve $\alpha(p)$ identified to a point, which is mapped to $p \in \bar{\Sigma}$. Choose and fix a corresponding quotient map $q: F \rightarrow \bar{\Sigma}$. Let $i: F \rightarrow M$ be the composition $i=\iota \circ q$. Observe that $\Sigma$ can be identified with a subset of $F$ so that $q$ is the identity map on $\Sigma$ and $i=\iota$ on $\Sigma$.

We next construct diffeomorphisms $\varphi_{j}: F_{j} \rightarrow F$. These will be extensions of the diffeomorphisms $\psi_{j}: \kappa_{j} \rightarrow \Theta_{j} \subset \Sigma$ of Section 5 using the identification of $\Sigma_{j}$ with a subset of $F_{j}$.

We recall that the set $\Theta_{j}$ is the complement of a union of horocyclic neighbourhoods of cusps. Thus, there are punctured discs $\Delta(j ; z) \subset \Sigma, z \in S(\Sigma)$ and $\Delta^{ \pm}(j ; p) \subset \Sigma, p \in \Lambda(\Sigma)$ so that $\Theta_{j}$ is the complement of the interiors of the sets $D(j ; z)$ and $D^{ \pm}(j ; p)$. Without loss of generality we assume that $k \geq j$ implies that $\Delta(k ; z) \subset \Delta(j ; z)$ and $\Delta^{ \pm}(k ; p) \subset \Delta^{ \pm}(j ; p)$ for all $z$ and $p$.

We shall make use of the constructions of Lemmas 8.1 and 8.2 . As the sets $\Theta_{j}$ form an exhaustion of $\Sigma$, for each fixed $z \in S(\Sigma)$ or $p \in \Lambda(\Sigma)$, the intersection of the corresponding punctured discs $\Delta(j ; p)$ and $\Delta^{ \pm}(j ; p)$ is empty. It follows as in the proofs of Lemmas 8.1 and 8.2 that if $D_{k}(z)$ and $B_{k}(p)$ denote the corresponding discs and annuli in $F_{k}$ for $k$ sufficiently large, then the diameters of these sets are bounded above by $\delta_{j}$ so that $\delta_{j} \rightarrow 0$ as $k \rightarrow \infty$.

We take $\varphi_{j}$ to be an extension of $\psi_{j}$ so that the complement of the set $\kappa_{j} \subset F_{j}$ is mapped to the complement of the set $\Theta_{j} \subset \Sigma \subset F$. Any compact set $K \subset \Sigma$ is contained in $\Theta_{j}$ for $j$ sufficiently large. Hence, $i_{j} \circ \varphi^{-1}=i_{j} \circ \psi_{j}^{-1}$ converges to $i=\iota: \Sigma \rightarrow M$. Finally, for the points of $S(F)$ and points on $\alpha_{i}$, by the continuity of the extension of $\iota: \Sigma \rightarrow M$ to $\bar{\Sigma}$, we see that $i_{j} \circ \varphi^{-1}: F \rightarrow M$ converges to $i=\iota \circ q: F \rightarrow M$.

\section{A Limiting Metric}

We continue to use the notation of the previous section. Using the diffeomorphisms $\varphi_{j}$, we can identify the surfaces $F_{j}$ with $F$. Hence the pullback metrics on the surface $F_{j}$ give Riemannian metrics on $F$ which have associated distance functions $d_{j}$. We shall construct a limiting pseudo-metric $d$ on the surface $F$. Recall that this is a symmetric function $d: F \times F \rightarrow[0, \infty)$ that satisfies the triangle inequality but not in general the positivity condition for metrics. 
Lemma 10.1. On passing to a subsequence, the metrics $d_{j}$ converge uniformly on $F$ to a pseudo-metric d.

Proof. Let $g_{0}$ denote the fixed hyperbolic metric on $\Sigma$ and $d_{0}$ the distance function of this metric. Our first step is to show that the distance functions $d_{j}$ converge on $\Sigma$.

Lemma 10.2. The family of function $d_{j}: \Sigma \times \Sigma \rightarrow \mathbb{R}$ is equicontinuous on every compact subset $K$ of $\Sigma \times \Sigma$, where we consider the product metric obtained from $d_{0}$ on $\Sigma \times \Sigma$.

Proof. Showing equicontinuity on the product is equivalent to showing that given $\eta>0$ there is a $\delta>0$ so that for pairs $\left(p_{i}, q_{i}\right), i=1,2$, such that $d_{0}\left(p_{1}, p_{2}\right)<\delta$ and $d_{0}\left(q_{1}, q_{2}\right)<\delta$, we have $\left|d_{j}\left(p_{1}, q_{1}\right)-d_{j}\left(p_{2}, q_{2}\right)\right|<\eta$. Note that by the triangle inequality,

$$
\left|d_{j}\left(p_{1}, q_{1}\right)-d_{j}\left(p_{2}, q_{2}\right)\right|<d_{j}\left(p_{1}, p_{2}\right)+d_{j}\left(q_{1}, q_{2}\right) .
$$

Hence it suffices to show that we can choose $\delta$ so that $d_{0}\left(p_{1}, p_{2}\right)<\delta$ implies $d_{j}\left(p_{1}, p_{2}\right)<\eta$. But this follows from Lemma 7.1, where we have shown the uniform Lipshitz property of the functions $d_{j}$ on compact subsets of $\Sigma$.

We shall apply the above to the compact sets $\Theta_{j} \in \Sigma=F$ of the previous section, which exhaust $\Sigma$. By the above lemma the distance functions $d_{k}$ are uniformly Lipshitz on $\Theta_{j}$ for each $j$. Hence, we can iteratively pass to subsequences and use a diagonal sequence to ensure that the metrics $d_{k}$ have a limit $d$ with convergence uniform on each set $\Theta_{j} \times \Theta_{j}$. More precisely, we can ensure that if $p, q \in \Theta_{j}$ and $k \geq j$, then $\left|d_{k}(p, q)-d(p, q)\right|<\delta_{j}^{\prime}$ with $\delta_{j}^{\prime} \rightarrow 0$ as $j \rightarrow \infty$.

As $d$ is the limit of metrics, it is a pseudo-metric on $\Sigma$. Let $\bar{\Delta}(j ; z)$ denote the closure of $\Delta(j ; z)$ in $F$ and $\bar{\Delta}(j ; p)$ denote the closure of $\Delta^{+}(j ; p) \cup \Delta^{-}(j ; p)$ in $F$. Observe that these sets are identified with sets $D_{j}(z)$ and $B_{j}(p)$ under the map $\varphi_{j}$ and hence have diameter at most $\delta_{j}$ in the metric $d_{k}$ for $k$ sufficiently large (as in the previous section). Hence the diameter of the sets $\bar{\Delta}(j ; z)$ and $\bar{\Delta}(j ; p)$ in the pseudo-metric $d$ is at most $\delta_{j}$.

We shall extend $d$ to $F$ by continuity. First consider the case where $p \in \Sigma$ and $q \in \alpha(p)$ for some $p \in \Lambda(\Sigma)$. Consider a sequence $q_{i}$ in $\Sigma$ converging to $q$. Without loss of generality we can assume that $q_{j} \in \bar{\Delta}(j ; p)$ for some $p$. We claim that the sequence $d\left(p, q_{j}\right)$ is Cauchy. For $k \geq j$, as $q_{k} \in \bar{\Delta}(k ; p) \subset \bar{\Delta}(j ; p)$ and the diameter of $\bar{\Delta}(j ; p)$ in the pseudo-metric $d$ is at most $\delta_{j}, d\left(q_{j}, q_{k}\right) \leq \delta_{j}$. Hence, by the triangle inequality,

$$
\left|d\left(p, q_{j}\right)-d\left(p, q_{k}\right)\right| \leq d\left(q_{j}, q_{k}\right) \leq \delta_{j} .
$$

It follows that the sequence $d\left(p, q_{j}\right)$ is Cauchy and hence converges to a limit which we define to be $d(p, q)$. Observe that if $q_{j}^{\prime}$ is another sequence in $\bar{\Delta}(k ; p)$, then as above $\left|d\left(p, q_{j}\right)-d\left(p, q_{j}^{\prime}\right)\right|<\delta_{j}$. Hence the limit is well-defined.

We can similarly define $d(p, q)$ if $q \in S(\Sigma)$. In case neither $p$ nor $q$ are in $\Sigma$, we use sequences $p_{j}^{\prime}$ and $q_{j}^{\prime}$ in $\Sigma$ converging to these points. As above we get Cauchy sequences with limit independent of the choice of sequence.

We finally show that the convergence is uniform on all of $F$. Suppose now that $p, q \in F$ are arbitrary. We shall find a uniform upper bound for the quantity $\left|d_{j}(p, q)-d(p, q)\right|$. Suppose $p, q \in \Theta_{j}$; then as above $\left|d_{j}(p, q)-d(p, q)\right| \leq \delta_{j}^{\prime}$. Otherwise, one of $p$ and $q$ is in the interior of some set of the form $\bar{\Delta}(j ; p)$ or 
$\bar{\Delta}(j ; z)$. We consider the case when $p \in \Theta_{j}$ and $q \in \bar{\Delta}(j ; p)$, as the other cases are similar.

Let $q^{\prime}$ be a point in the boundary $\partial \bar{\Delta}(j ; j)=\bar{\Delta}(j ; p) \cap \Theta_{j}$. Then as above, $\left|d_{j}\left(p, q^{\prime}\right)-d\left(p, q^{\prime}\right)\right|<\delta_{j}^{\prime}$. Further, as the set $\bar{\Delta}(j ; p)$ has a diameter at most $\delta_{j}$ in the (pseudo)metrics $d_{j}$ and $d, d\left(q, q^{\prime}\right) \leq \delta_{j}$ and $d_{j}\left(q, q^{\prime}\right) \leq \delta_{j}$.

By the triangle inequality, it follows that

$$
\left|d_{j}(p, q)-d(p, q)\right| \leq 2 \delta_{j}+\delta_{j}^{\prime} .
$$

This shows that we have uniform convergence of the metrics $d_{k}$ to $d$ on all of $F$.

As the final step in the proof of Theorem 2.1 we see that the limiting map $i: F \rightarrow M$ is Lipschitz with respect to the pseudo-metric $d$. Note that, for the distance functions $d_{j}$ corresponding to the pullback metrics $\left(i_{j} \circ \varphi_{j}^{-1}\right)^{*}(g)$ on $F$, the maps $i_{j} \circ \varphi_{j}^{-1}$ are distance decreasing (by definition of pullback Riemannian metrics). By taking limits, we see that $i: F \rightarrow M$ is distance decreasing for the pseudometric $d$, and hence Lipschitz.

This completes the proof of Theorem 2.1.

In the sequel, we shall use the notation

$$
\left|d_{j}-d\right|:=\sup _{p, q \in F}\left|d_{j}(p, q)-d(p, q)\right| .
$$

\section{Fractal dimension of the limit}

We have constructed a limiting metric $d$ on the surface $F$. In this section, we show that this metric has fractal dimension two and has finite, non-zero area in an appropriate sense. This gives a proof of Theorem 2.3

Now we come to the main lemma of this section.

Lemma 11.1. Let $(F, g)$ be a compact Riemannian 2-manifold with sectional curvature $K \leq K_{0}$. For any $p \in F$ and $0<\delta<\frac{1}{\sqrt{3 K_{0}}}$, if

$$
\operatorname{vol}(B(p, \delta))>C \delta^{2}
$$

then there is a $\delta^{\prime}<\delta$ with

$$
\int_{B\left(p, \delta^{\prime}\right)} K d v \leq 2 \pi-\frac{C}{2}
$$

Proof. By making an arbitrarily small $C^{2}$ perturbation of $g$ we can assume that it is real-analytic. Recall that $\frac{\pi}{3 \sqrt{K_{0}}}$ is the uniform lower bound on the conjugate radius given by Lemma 3.2 . Hence

$$
\tilde{g}:=\exp ^{*}(g)
$$

is a Riemannian metric on the (Euclidean) ball $B(0, \delta)$ in $T_{p} M$. Also,

$$
\exp _{p}: \text { Int }(U \cap B(0, \delta)) \rightarrow \text { Int } B(p, \delta)
$$

is a diffeomorphism, where $U=U_{p}$ as in Section 3.5 Note that $U$ is star-shaped with respect to $0 \in T_{p} M$.

If we let $P=U \cap B(0, \delta)$, then by Lemma $3.7 \partial P$ is a piecewise-smooth 1manifold. In fact we can write

$$
\partial P=L_{1} \cup \ldots \cup L_{k} \cup S_{1} \cup \ldots \cup S_{l},
$$


where each $S_{i}$ is a smooth closed segment contained in the circle $S(0, \delta)$ and each $L_{i}$ is a smooth closed segment contained in the cut-locus $\partial U$. We refer to $L_{i}$ and $S_{i}$ as sides of $P$ and the non-smooth points of $\partial P$ as vertices of $P$. We can assume that a side intersects another side in at most one point.

By changing $\delta$ slightly, we can assume that vertices of $\partial P$ are either common points of a side $L_{i}$ and a side $S_{j}$ or two sides $L_{i}$ and $L_{j}$.

By the definition of the cut-locus, for each $L_{i}$ there is at least one $L_{j}, j \neq i$, such that $\exp _{p}\left(L_{i}\right)=\exp _{p}\left(L_{j}\right)$. For any such pair $L_{i} \cap L_{j}=\phi$, since we know that $\exp _{p}$ is a local diffeomorphism on $B(0, \delta)$.

Lemma 11.2. We have the following:

(1) $\sum_{i=1}^{k} \int_{L_{i}} \kappa=0$.

(2) The angle between any two consecutive sides of $P$ is positive.

Proof. As $\delta$ is less than the conjugacy radius, we have Gauss normal coordinates on $P$ which we denote by $r$ and $\theta$ as usual, with the coordinates of a point $x$ denoted by $r(x)$ and $\theta(x)$.

Let $L_{j}$ be a segment as above parametrised by a function $\alpha(s)$, with $\alpha(0)$ a point in the interior of $L_{j}$. Then, as $L_{j}$ is in the boundary of $P$, there is a subsegment of some segment $L_{i}$, parametrised by $\beta(s)$, so that $\alpha(s)$ and $\beta(s)$ have the same image $c(s)$ under the exponential map and $d(\alpha(s), p)=d(\beta(s), p)$, i.e. $r(\alpha(s))=r(\beta(s))$, in a neighbourhood of $s=0$. The images of the radial vectors joining $p$ to $\alpha(s)$ and $\beta(s)$ form geodesics $\gamma_{s}$ and $\xi_{s}$ of the same length joining $p$ to $c(s)$.

By differentiating $r(\alpha(s))=r(\beta(s))$ and considering images in $F$, we see that the inner products of $\alpha^{\prime}(0)$ and $\beta^{\prime}(0)$ with the respective radial vectors are equal. As $\alpha(s)$ and $\beta(s)$ have the same image on a neighbourhood of $0, \alpha^{\prime}(0)$ and $\beta^{\prime}(0)$ have the same norm. It follows that the angles made by the vectors $\alpha^{\prime}(0)$ and $\beta^{\prime}(0)$ with the respective unit radial vectors are the same. On passing to the image, we see that the geodesics $\gamma_{0}$ and $\xi_{0}$ make the same angle with $c^{\prime}(0)$ at the point $c(0)$.

It follows that $\gamma_{0}$ and $\xi_{0}$ must approach $c(0)$ on opposite sides - otherwise they would have a common point and direction and hence coincide. In particular, there are exactly two points in $\partial P$ that map to a smooth (even $C^{1}$ ) point on the cutlocus, for if there were at least three points, two would be on the same side. It follows that the segments $L_{j}$ are identified in pairs, with the interior of $P$ mapping to opposite sides of the image.

We deduce that

$$
\sum_{i=1}^{k} \int_{L_{i}} \kappa=0
$$

where $\kappa$ denotes geodesic curvature. This is because the terms that correspond to $L_{i}$ and $L_{j}$ mapping to the same segment have equal magnitude (as their image is equal) and opposite signs (as the interior of $P$ maps to opposite sides of the image).

We next see that the internal oriented angle $\theta_{i}$ between any two consecutive closed segments in $U \cap B(0, \delta)$ is positive. Observe that as $P$ is a star convex region with boundary piecewise smooth, we can parametrise $\partial P$ by angle using a function $r(\theta)$. This is smooth wherever $\partial P$ is smooth. The left and right derivatives $r_{ \pm}^{\prime}(\theta)$ exist at all points. All internal oriented angles are positive if and only if for every non-smooth point (i.e., vertex) $\theta, r_{-}^{\prime}(\theta) \geq r_{+}^{\prime}(\theta)$. 
First consider the case when a vertex of $\partial P$ is between an edge of the form $L_{i}$ and one of the form $S_{j}$. By construction, on $S_{j}$ we have $r(\theta)=\delta$ and on $L_{i}$ we have $r(\theta) \leq \delta$. It is immediate that $r_{-}^{\prime}(\theta) \geq r_{+}^{\prime}(\theta)$.

Next, consider a vertex $v$ between segments $L_{i_{1}}$ and $L_{i_{2}}$, parametrised by $\alpha_{1}(s)$, $s \leq 0$ and $\alpha_{2}(s), s \geq 0$, respectively. By the above, there are edges $L_{j_{1}}$ and $L_{j_{2}}$ that can be parametrised by curves $\beta_{1}$ and $\beta_{2}$, with the images of $\alpha_{i}$ and $\beta_{i}$ coinciding and $d\left(p, \alpha_{i}(s)\right)=d\left(p, \beta_{i}(s)\right)$.

We see that the curves $\alpha_{i}$ and $\beta_{i}$ can be extended so that their domain of definition includes a neighbourhood of the origin and with the images of $\alpha_{i}$ and $\beta_{i}$ coinciding and $d\left(p, \alpha_{i}(s)\right)=d\left(p, \beta_{i}(s)\right)$. Without loss of generality, we prove this for $\alpha=\alpha_{1}$ and $\beta=\beta_{1}$. Namely, as $\delta$ is less than the conjugacy radius, the exponential map gives diffeomorphisms from neighbourhoods $U_{\alpha}$ and $U_{\beta}$ of $\alpha(0)$ and $\beta(0)$ to a neighbourhood $V$ of their image $v$. The images of the coordinate function $r$ under these diffeomorphisms gives coordinate functions $r_{\alpha}$ and $r_{\beta}$. The condition $d\left(p, \alpha_{i}(s)\right)=d\left(p, \beta_{i}(s)\right)$ is equivalent to $r_{\alpha}=r_{\beta}$.

The gradients of the functions $r_{\alpha}$ and $r_{\beta}$ at $v$ are unit vectors along the geodesic segments from $p$ to the vertex $v$ that are the images of the radial vectors to $\alpha(0)$ and $\beta(0)$. As these geodesics do not coincide, the gradients do not coincide and hence the gradient of $r_{\alpha}-r_{\beta}$ is non-zero. It follows that the set $r_{\alpha}=r_{\beta}$ is a manifold near $v$. Taking inverse images under the diffeomorphisms from $U_{\alpha}$ and $U_{\beta}$ to $V$ gives the required smooth extensions of $\alpha$ and $\beta$.

We now consider these extensions of $\alpha_{1}$ and $\alpha_{2}$. By the definition of the cutlocus, it follows that for $s>0, r\left(\alpha_{2}(s)\right) \leq r\left(\alpha_{1}(s)\right)$. As $\alpha_{1}(s)$ is smooth at 0 , we deduce that $r_{-}^{\prime}(\theta) \geq r_{+}^{\prime}(\theta)$ if $\theta=\alpha(0)$ is the given vertex. Thus, the angles are positive in this case, too. This completes the proof of the lemma.

We now return to the proof of Lemma 11.1. Since $\delta<\frac{\pi}{3 \sqrt{K_{0}}}$, we can compare the Riemannian manifold $(B(0, \delta), \tilde{g})$ with the round 2 -sphere of radius $\frac{1}{\sqrt{K_{0}}}$ (the advantage of working with $B(0, \delta)$ in $T_{p} M$ rather than $B(p, \delta)$ in $M$ is that the injectivity radius of $B(0, \delta)$ with the pull-back metric $\tilde{g}$ is $\delta)$. If $\kappa$ denotes the mean-curvature function on $S(0, \delta)$, then

$$
\kappa \geq \sqrt{K}_{0} \frac{\cos \left(\sqrt{K}_{0} \delta\right)}{\sin \left(\sqrt{K}_{0} \delta\right)} \quad \frac{1}{2 \delta} \quad \text { on } \quad S(0, \delta)
$$

Now we can apply the Gauss-Bonnet theorem to get

$$
\begin{aligned}
\int_{B(p, \delta)} K & =\int_{U \cap B(0, \delta)} \tilde{K} \\
& =2 \pi \chi(B(0, \delta))-\sum_{i=1}^{k} \int_{L_{i}} \kappa-\sum_{j=1}^{l} \int_{S_{j}} \kappa-\sum_{i=1}^{k} \theta_{i} \\
& \leq 2 \pi-\sum_{j=1}^{l} \int_{S_{j}} \kappa \\
& \leq 2 \pi-\frac{l(\delta)}{2 \delta}
\end{aligned}
$$


where $\tilde{K}$ is the Gaussian curvature of $\tilde{g}$ and

$$
l(\delta)=\sum_{j=1}^{l} \operatorname{length}\left(S_{j}\right) .
$$

Note that we have used Lemma 11.2 (proved earlier) in going from line 2 to line 3 above. The area of $B(p, \delta)$ is given by

$$
\operatorname{vol}(B(p, \delta))=\operatorname{vol}(U \cap B(0, \delta))=\int_{0}^{\delta} l(s) d s .
$$

Hence if $\operatorname{vol}(B(p, \delta)) \geq C \delta^{2}$ for some $C$, then there exists $\delta^{\prime} \leq \delta$ with

$$
l\left(\delta^{\prime}\right) \geq C \delta .
$$

By (4), we would have for this $\delta^{\prime}$

$$
\int_{B\left(p, \delta^{\prime}\right)} K \leq 2 \pi-\frac{l\left(\delta^{\prime}\right)}{2 \delta^{\prime}} \leq 2 \pi-\frac{C}{2} .
$$

This completes the proof of Lemma 11.1

Corollary 11.3. Let $(F, g)$ be a compact Riemannian 2-manifold with sectional curvature $K \leq K_{0}$ and area $A$ satisfying $a_{0} \leq A \leq A_{0}$. Suppose that $S=\left\{x_{1}, \ldots, x_{l}\right\}$ is a $\delta$-net with $\delta<\frac{1}{\sqrt{2 K_{0}}}$.

Then, for any $x_{i} \in S$, we have

$$
\operatorname{vol} B\left(x_{i}, \delta\right) \leq C_{0} \delta^{2}
$$

where

$$
C_{0}=8 \pi^{2}(1-\chi(F))+4 \pi K_{0} A_{0} .
$$

Hence it follows that the cardinality of $S$ is at least $\frac{a_{0}}{C_{0}} \delta^{-2}$.

Proof. We will assume that at some point, say $x_{1}$,

$$
\operatorname{vol} B\left(x_{1}, \delta\right) \geq C_{0} \delta^{2},
$$

and we get a contradiction. The Gauss-Bonnet theorem applied to $F$ along with Lemma 11.1 gives

$$
\begin{aligned}
2 \pi \chi(F) & =\int_{B\left(x_{1}, \delta^{\prime}\right)} K d v+\int_{F-B\left(x_{1}, \delta^{\prime}\right)} K d v \\
& \leq 2 \pi-\frac{C_{0}}{2}+K_{0} A_{0} .
\end{aligned}
$$

This gives

$$
C_{0}<4 \pi(1-\chi(F))+K_{0} A_{0}
$$

a contradiction.

Let $F$ be a surface in $M$ with the given bounds on mean curvature, genus and area bounded above by $A_{0}$. Then the sectional curvature of $F$ is bounded above. Next we see that there is a lower bound $a_{0}$ on the area of $F$ depending only on the geometry of $M$ and the given bounds on $F$. This allows us to apply the above corollary uniformly.

Lemma 11.4. There is a constant $a_{0}$ depending only on the geometry of $M$ and the bound on the mean curvature of $F$ such that the area of $F$ is at least $a_{0}$. 
Proof. This follows from Theorem 4.2 applied for an arbitrary value of $\epsilon$.

We next show that there is a lower bound on the area of a ball of radius $\delta$, hence an upper bound on the size of a $\delta$-net.

Lemma 11.5. There is a constant $c>0$ such that for $\delta$ sufficiently small, the area of the ball of radius $\delta$ in $F$ around a point $p \in F$ is at least $c \delta^{2}$. As a consequence the size of a $\delta$-net is at most $A_{0} / c(\delta / 2)^{2}$.

Proof. Again, by using Theorem 4.2, we can deduce the bound on the size of the $\delta$-net. Note that for a $\delta$-net $S$, the balls of radius $\delta / 2$ centered around the points of $S$ are disjoint. Hence their total area is at most the area of $F$, which is in turn at most $A_{0}$. As the area of each of these balls is at least $c(\delta / 2)^{2}$, it follows that the cardinality of $S$ is at most $A_{0} / c(\delta / 2)^{2}$.

We conclude that the size of a $\delta$-net for the metric $d$ grows as $\delta^{-2}$ as $\delta \rightarrow 0$.

Theorem 11.6. There are constants $0<b<B<\infty$ such that, for $\delta$ sufficiently small, the size of a $\delta$-net $S$ for the pseudometric d satisfies

$$
b \delta^{-2} \leq|S| \leq B \delta^{-2} .
$$

Proof. Suppose $\delta>0$ is sufficiently small and $S$ is a $\delta$-net for $d$, i.e., a maximal set so that all pairwise distances are at least $\delta$. Let $j$ be such that $\left|d_{j}-d\right|<\delta / 2$. Then for $p, q \in S, p \neq q$, we have $d_{j}(p, q) \geq \delta / 2$. Hence $S$ is contained in a $\delta / 2$-net $S^{\prime}$ for the metric $d_{j}$. However, Lemma 11.5 gives an upper bound of the form $B \delta^{-2}$ for the cardinality of $S^{\prime}$, and hence of $S$.

Next, let $T$ be a $3 \delta$-net for the metric $d_{j}$. We claim that the cardinality of $T$ is at most that of $S$. First observe that as $S$ is a $\delta$-net, if $x \in F$, then for at least one $p=p(x)$ in $S, d(x, p) \leq \delta$. If $x \in S$, this is obvious, otherwise by considering $S \cup\{x\}$ we get a contradiction to the maximality. As $\left|d_{j}-d\right|<\delta / 2$, it follows that $d_{j}(x, p(x))<3 \delta / 2$.

For each point $q \in T$, choose and fix $p(q)$ as above. This gives a function $p: T \rightarrow S$.

Lemma 11.7. $p: T \rightarrow S$ is injective.

Proof. Suppose $p(q)=p\left(q^{\prime}\right)=p$. Then we have seen that $d_{j}(q, p) \leq 3 \delta / 2$ and $d_{j}\left(q^{\prime}, p\right)<3 \delta / 2$. By the triangle inequality, $d_{j}\left(q, q^{\prime}\right)<3 \delta$, contradicting the hypothesis that $T$ is a $3 \delta$-net for the metric $d_{j}$.

It is immediate that the cardinalities of $S$ and $T$ satisfy $|T| \leq|S|$. But Corollary 11.3 gives a lower bound of the form $b \delta^{-2}$ on $|T|$, hence on the cardinality of $S$.

A coarse notion of area (and volume) and the corresponding notion of dimension, the so-called fractal dimension, can be defined in terms of $\delta$-nets. Namely, let $(X, d)$ be a metric space. For $\delta>0$, let $n(\delta)$ be the minimum number of balls of radius $\delta$ that cover $X$. For $s .0$, define the $s$-dimensional volume by

$$
V^{s}(X)=\limsup _{\delta \rightarrow 0} n(\delta) \delta^{s}
$$

It is an immediate consequence of Theorem 11.6 that the 2-dimensional volume, in the above sense, of $F$ with the metric $d$ is a finite, positive number. Further, for $s<2$ the $s$-dimensional volume is zero and for $s>2$ it is infinite. Thus, the 
limiting metric on the surface is a metric of fractal dimension two and of finite, positive 2-dimensional volume.

The fractal dimension is closely related, but not equal, to the Hausdorff dimension. In particular, it is a capacity rather than a measure - we have finite but not countable additivity. For example, if $X$ is the set $\mathbb{Q} \cap[0,1]$ of rational numbers in $[0,1]$, then $V^{1}(X)=1$, but the 1-dimensional Hausdorff measure of $X$ is zero.

It is easy to deduce from the above that the Hausdorff dimension of $F$ is at most 2. However, we do not know whether the Hausdorff dimension must be two. We remark that the metric $d_{j}$ is not in general bi-Lipshitz to the pseudo-metric $d$. As for pairs of distinct points $p, q$ in a circle $\alpha^{i}$ in $F$ as above (if there is at least one such circle), $d(p, q)=0$ but $d_{j}(p, q) \neq 0$.

\section{Appendix A: Bounds on curvature and conjugate radius}

Lemma (see Lemma 3.1). Let $F$ be an embedded surface in a Riemannian nmanifold $(M, g)$ with mean curvature bounded above by $H_{0}$. There is a constant $K_{0}=K_{0}\left(M, g, H_{0}\right)$ so that the sectional curvature of $F$ is bounded above by $K_{0}$.

Proof. Let $\tilde{\nabla}$ and $\nabla$ denote the Riemannian connections of $M$ and F. Fix $p \in F$ and let $N_{1}, \ldots, N_{n-2}$ be unit normal vector fields defined in a neighbourhood of $p$. Then the second fundamental form $B$ is given by

$$
B(X, Y):=\widetilde{\nabla}_{X} Y-\nabla_{X} Y
$$

and can be written as

$$
\left.B(X, Y)=\sum_{i=1}^{n-2}\left\langle B_{i}(X), Y\right) N_{i}\right\rangle, \quad X, Y \in T_{p} F
$$

where the symmetric linear operators $B_{i}: T_{p} F \rightarrow T_{p} F$ are given by $B_{i}(X)=$ $-\left(\widetilde{\nabla}_{X} N_{i}\right)^{T}$. The mean curvature field is given by

$$
H=\sum_{i=1}^{n-2} \operatorname{Tr}\left(B_{i}\right) N_{i}
$$

If $k_{a}$ is the sectional curvature of $M$ along the tangent plane $T_{p} F$, then, by the Gauss-Codazzi formula, the sectional curvature of $F$ at $p$ is given by

$$
k=k_{a}+\sum_{i=1}^{n-2} \operatorname{Det}\left(B_{i}\right)
$$

Fix $i$ for now. Let $\kappa_{1}$ and $\kappa_{2}$ denote the eigenvalues of $B_{i}$. Since

$$
|H|^{2}=\sum_{i=1}^{n-2} \operatorname{Tr}\left(B_{i}\right)^{2},
$$

we have $\left|\kappa_{1}+\kappa_{2}\right| \leq|H| \leq H_{0}$.

Hence

$$
\operatorname{Det}\left(B_{i}\right)=\kappa_{1} \kappa_{2}=\frac{\left(\kappa_{1}+\kappa_{2}\right)^{2}-\left(\kappa_{1}-\kappa_{2}\right)^{2}}{4} \leq \frac{H_{0}^{2}}{4} .
$$

Since $M$ is compact, there is an upper bound $K_{M}$ on $k_{a}$. It follows that the sectional curvature of $F$ is bounded above by $K_{0}=K_{M}+(n-2) H_{0}^{2} / 4$. 
Lemma (see Lemma 3.2). Let $(F, g)$ be a complete Riemannian 2-manifold with sectional curvature bounded above by $K_{0}$. Then the conjugate radius at any $p \in F$ is at least $R:=\frac{\pi}{3 \sqrt{K_{0}}}$. Moreover, if we write

$$
\exp ^{*}(g)=d r^{2}+f^{2}(r, \theta) d \theta^{2}
$$

for polar coordinates $(r, \theta)$ on $T_{p} F$ and $r<R$, then $f(r, \theta)$ is increasing as a function of $r$ and $f(r, \theta)>r / 2$ for all $\theta$.

Proof. Fix polar coordinates $(r, \theta)$ on $T_{p} F$; exp will denote $\exp _{p}$. We know that

$$
\exp _{*}\left(\frac{\partial}{\partial r}\right)=\frac{\partial}{\partial r} \text {. }
$$

Let

$$
J_{\theta}(r)=\left.\exp _{*}\right|_{(r, \theta)}\left(\frac{\partial}{\partial \theta}\right), \quad f(r, \theta)=\left\|J_{\theta}(r)\right\| .
$$

Note that

$$
\exp ^{*}(g)=d r^{2}+f^{2}(r, \theta) d \theta^{2} .
$$

Fix $\theta>0$ and regard $f$ as a function $r$ alone. Let $T>0$ be the smallest value of $T$ such that $J_{\theta}(T)=0$. Then $f$ is smooth on $[0, T)$. Assume that $r \in(0, T]$. Since $J$ is a Jacobi field, we have

$$
K(r, \theta) f(r, \theta)=-\frac{\partial^{2} f(r, \theta)}{\partial r^{2}},
$$

where $K$ denotes the Gaussian curvature of $F$. Therefore

$$
f^{\prime \prime}+K_{0} f \geq 0 .
$$

The above inequality combined with

$$
f(0)=0, \quad f^{\prime}(0)=1 \text { and } f \geq 0
$$

implies that

for

Hence

$$
\sin \left(\sqrt{K_{0}} r\right) f^{\prime}(r)-\sqrt{K_{0}} \cos \left(\sqrt{K_{0}} r\right) f(r) \geq 0
$$

$$
r \in I=\left[0, \frac{\pi}{2 \sqrt{K}_{0}}\right] .
$$

$$
f^{\prime}(r) \geq \sqrt{K_{0}} \cot \left(\sqrt{K_{0}} r\right) f(r) \geq 0
$$

on $I$. This implies that $f>0$ on $I$, since $f$ is non-decreasing on $I$ and $f(r)=0$ if and only if $f^{\prime}=0$ on $(0, r)$, which would contradict $f^{\prime}(0)=1$.

Integrating (5), we get

$$
f(r) \geq \frac{f(t)}{\sin \left(\sqrt{K}_{0} t\right)} \sin \left(\sqrt{K}_{0} r\right)
$$

for $0<t<r<\frac{\pi}{2 \sqrt{K_{0}}}$.

Letting $t \rightarrow 0$,

$$
f(r) \geq \frac{1}{\sqrt{K}_{0}} \sin \left(\sqrt{K}_{0} r\right)>\frac{r}{2}
$$

for $0<r<\frac{\pi}{3 \sqrt{K}_{0}}$.

Hence we can take $R=\frac{\pi}{3 \sqrt{K_{0}}}$. 


\section{Appendix B: Lifting Discs Under THE EXPONENTIAL MAP}

In this section, we prove Lemma 3.3 which allows us to lift discs to the tangent space.

Lemma (see Lemma 3.3). Let $\iota: B \rightarrow(F, g)$ be an immersion of a disc into a complete Riemannian 2-manifold $(F, g)$ with sectional curvature bounded above by $K_{0}$. Suppose that for the pullback metric $i^{*} g$, the length of $\gamma=\partial B$ and the distance of a point in $B$ to $\gamma$ are both bounded above by $\epsilon<R / 10$, where $R=\frac{\pi}{3 \sqrt{K_{0}}}$. Then for $x=\iota(y)$ in the image of $B$, there is a lift $\tilde{\iota}$ of $\iota$ to the tangent space $T_{x} F$ so that $\iota=\exp _{x} \circ \tilde{\iota}$. Furthermore, the lift can be chosen so that $\tilde{\iota}(y)$ is the origin.

Proof. Recall (Lemma 3.2) that exp is an immersion on a ball of uniform radius $R$ in $T_{p} F$ for any $p \in F$. Hence $\exp _{x}^{*}\left(g_{j}\right)$ is a metric on this ball and the injectivity radius at 0 is at least $R$. The tangent space $T_{x} F$ is a vector space with an inner product with origin identified with $x$. We seek lifts with respect to the exponential $\operatorname{map} \exp _{x}: T_{x} F \rightarrow F$.

By the inverse function theorem, Lemma 3.2 yields the following.

Lemma 13.1. There is a constant $\delta>0$ such that given any point $\xi=\exp _{x}(z)$, with $z \in B(0,9 \epsilon) \subset T_{x} F$, there is a map $\exp _{z}^{-1}: B(\xi, \delta) \rightarrow T_{x} F$ with $\exp _{x} \circ \exp _{\xi}^{-1}$ the identity map and $\exp _{z}^{-1}(\xi)=z$.

Thus, the exponential map is invertible on sets of diameter less than $\delta$ containing a point in the image of $B(0,9 \epsilon)$. Note that $\delta$ is not universal. However, none of the constants in the Schwarz lemma depend on $\delta$. Observe that as the metric on the domain $B$ of $i$ is the pullback metric, a set of diameter at most $\delta$ in $B$ has image of diameter at most $\delta$.

We shall construct a lift on the $\operatorname{disc} B$ by inductively lifting sets $b_{i}$ of small diameter, as in the proof of the homotopy lifting theorem in Algebraic Topology. However, we need to ensure that at each stage the lift remains within $B(0,9 \epsilon)$ to continue the process. In our situation we can indeed choose such sets $b_{i}$ using a geometric argument, making use of the fact that $\partial B(\gamma)$ has length less than $\epsilon$.

Lemma 13.2. There is a sequence of smooth balls $b_{i}$, each of which has diameter at most $\epsilon$, so that if $B_{j}=\bigcup_{i=1}^{j} b_{i}$ and $B_{0}$ is a single point $B_{0}=\left\{y_{0}\right\}$,

(1) The set $B_{j} \cap b_{j+1}$ is connected and non-empty for $j \geq 0$.

(2) The set $B_{j} \cap \partial B$ is connected and non-empty for each $j$.

(3) For each point $p \in B_{j}$, there is a path $\alpha$ contained in $B_{j}$ of length at most $2 \epsilon$ joining $p$ to $B_{j} \cap \partial B$.

Proof. We shall first construct discs $b_{i}$ with diameter at most $\delta$ and then re-order them to satisfy the condition of the lemma.

Consider the function $f: B \rightarrow \mathbb{R}$ given by the distance to the boundary. This is positive on the interior of $B$ and vanishes on the boundary. After a small perturbation, we can assume that this is Morse. Clearly the function $f$ has no local minima in the interior of $B$.

Thus, $f$ has finitely many critical points of index one and of index 2 . As there are no local minima, the descending manifold of each critical point of index 1 is a pair of arcs joining the critical point to the boundary. These partition the disc $B$ into closed subdiscs which we call basins. Each of these basins $P$ is the closure of the descending submanifold (i.e., the basin of repulsion of a critical point of index 


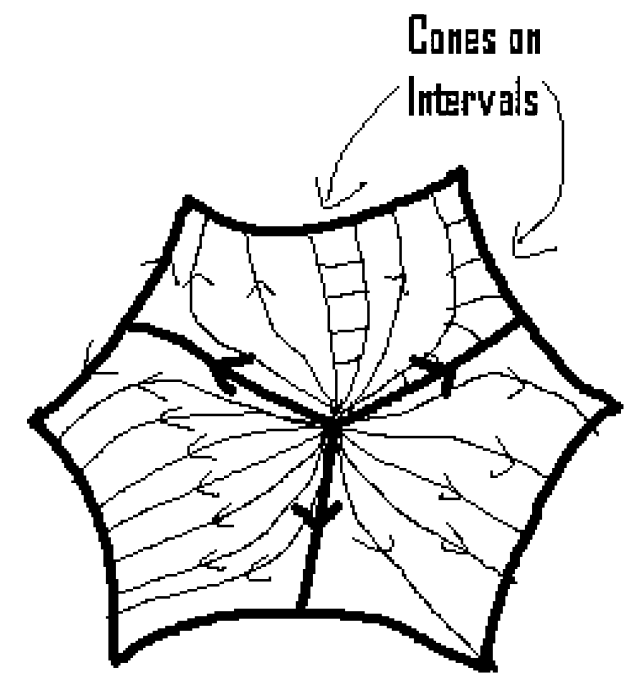

Figure 2. A basin with flows and cones

2 which we denote $O(P)$. We regard the gradient lines from the local maximum in a subdisc as radial lines (see Figure 2).

Now consider one such basin $P$. This is a polygon with $2 k$ sides for some $k \geq 0$, with alternate sides contained in the boundary of $B(\gamma)$ and alternate sides consisting of an index one critical point and the descending submanifolds of these.

Consider a closed interval $J$ in $P \cap \partial B$. We define the cone $C(J)$ to be the closure of the set of gradient lines that end in $J$. If $J$ is in the interior of $P \cap \partial B$ and has boundary points $a$ and $b$, then $C(J)$ is the region enclosed by $J$ together with the gradient lines joining $O(P)$ to $a$ and $b$ (see Figure 2).

Suppose next that one endpoint of $J$ is a vertex $v$ of $P$ and the other is an interior point $a$. The vertex $v$ is the limit of the gradient line joining an index-one critical point $x$ to $V$. The cone $C(J)$ is then the region enclosed by $J$, the gradient line from $O(P)$ to $a$, and an arc consisting of the closure of the gradient line from $O(P)$ to $x$ and the gradient line from $x$ to $v$.

In both of these cases, we can identify the cone with a sector in the circle, with gradient lines identified with radial lines. Using such an identification, the cone is foliated by lines transversal to the radial lines, namely those corresponding to lines of a fixed distance from the vertex of the sector, which we call longitudinal arcs $\lambda_{r}$. We call the point identified with the centre of the circle the centre of the cone and the arc $J$ the boundary arc.

By bounding the length of $J$ from above, we can ensure that the length of each $\operatorname{arc} \lambda_{r}$ is less than $\delta / 2$. We subdivide the boundary $\partial B$ into closed $\operatorname{arcs} J_{k}$ such that each arc $J_{k}$ is contained in some basin $P_{i_{k}}$ with at most one endpoint a vertex and with the lengths of the arcs $J_{k}$ sufficiently small to ensure that the corresponding longitudinal arcs in the cones have lengths at most $\delta / 2$. We get a partition of $B$ into corresponding subsets $C_{k}=C\left(J_{k}\right)$. 

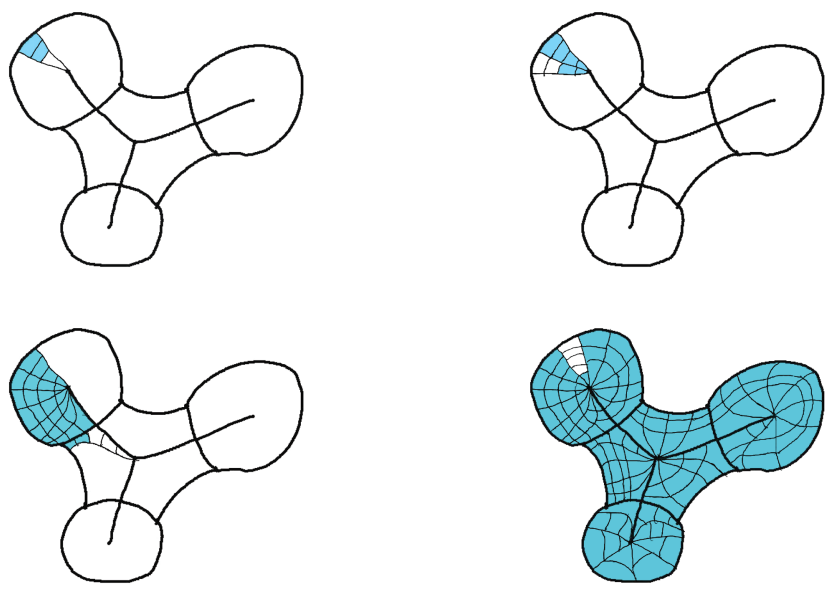

FigURE 3. Various stages of the exhaustion

We can further partition $C_{k}$ into regions between pairs of longitudinal arcs. For each $C_{k}$ we choose a collection of longitudinal arcs such that each of the regions between pairs of longitudinal arcs has diameter at most $\delta$. We shall call these regions squares (even though the region containing the centre is really a triangle).

This gives a partition of $B$ into discs $\delta$. After re-ordering, these discs will be the regions $b_{i}$. Observe that the regions in $C_{k}$ are naturally ordered starting with the region containing the centre and ending with the region containing the opposite arc. We shall use this as well as the opposite order. We shall often specify whether the first square is the one containing the centre or the boundary arc and consider the corresponding natural order.

We begin by ordering the $\operatorname{arcs} J_{k}$. Pick an $\operatorname{arc} J_{1}$ with both endpoints in the interior of an edge of a basin. Order the arcs cyclically beginning with the edge $J_{1}$. Let $P_{1}$ be the basin containing $J_{1}$ and let $y_{0}$ be a point in $J_{1}$.

We shall now order the squares $b_{i}$ (see Figure 3 showing $B_{j}$ at various stages). Consider the cone on the arc $J_{1}$ and let $b_{1}, b_{2}, \ldots, b_{l_{1}}$ be the regions of $C_{1}$ in the natural order so that $b_{1}$ contains $J_{1}$. By construction, for $i \leq l, B_{i} \cap \partial B=J_{1}$ and each point in $B_{i}$ is connected to $J_{1}$ by a radial line of length less than $\epsilon$.

Next, let $k$ be such that $J_{2}, \ldots, J_{k}$ are contained in $P$ and $J_{k+1}$ is not (this includes the case when there are only $k \operatorname{arcs} J_{i}$ ). We let $b_{l_{1}+1}$ be the square in $J_{2}$ containing the centre of $C_{2}$ and let $b_{l_{2}+2}, \ldots, b_{l_{2}}$ be the other squares in $C_{2}$ in the natural order. Observe that for $l_{1}<i \leq l_{2}$, each point in $B_{i}$ can be connected to the centre by a radial line of length at most $\epsilon$. The centre can in turn be connected to $J_{1} \subset \partial B \cap B_{i}$ by a radial line of length at most $\epsilon$. It is easy to see that the other claims also hold for the sets $b_{i}$ and $B_{i}$ constructed so far.

We now continue this process inductively, choosing $b_{l_{2}+1}$ to be the square of $C_{3}$ containing the centre and then choosing successive regions by the natural order. The same argument verifies the claims for these cases. In this manner, we can order all regions in the cones $J_{1}, \ldots, J_{k}$ to get $b_{1}, \ldots, b_{l}$.

Next consider (if we have not exhausted $B$ ) the cone $C_{k+1}$. We take the next square $b_{l+1}$ to be the square in $C_{k+1}$ that contains $J_{k+1}$. As before, the regions 
$b_{l+2}, \ldots$ will be the successive regions in $C_{k+1}$ in the natural order up to the region containing the centre. As for the first basin, for the successive interval $J_{k+2}, \ldots, J_{k^{\prime}}$ in the same basin as $J_{k+1}$, we take regions in successive intervals ordered starting with the region containing the centre.

The above constructions repeated inductively give an ordering of the regions $b_{i}$ satisfying all the claims.

Lemma 13.3. Given a set $B_{j}$ as above and two points $p, q \in B_{j}$, there is a path in $B_{j}$ of length at most $5 \epsilon$ joining $p$ to $q$.

Proof. The points $p$ and $q$ can be joined to points $p^{\prime}$ and $q^{\prime}$, respectively, in $B_{j} \cap \partial B$ by paths of length at most $2 \epsilon$. As $B_{j} \cap \partial B$ is connected and the length of $\partial B$ is at most $\epsilon, p^{\prime}$ and $q^{\prime}$ can be joined by a path of length at most $\epsilon$.

We now complete the proof of Lemma 3.3 . We inductively construct lifts $\tilde{\iota}_{j}$ on the sets $B_{j}$. First note that as $y_{0}$ and $x$ are in the set $B$ whose diameter is at most $3 \epsilon$, there is a point $z_{0} \in B(0,3 \epsilon) \subset T_{x} F$ with $\exp _{x}\left(z_{0}\right)=\iota\left(y_{0}\right)$. We define the map $\tilde{\iota}$ on $B_{0}$ by $\tilde{\iota}\left(y_{0}\right)=z_{0}$.

Next, we inductively construct a map $\tilde{\iota}_{j+1}$ on $B_{j+1}$ extending the given map on $B_{j}$. Doing this is equivalent to extending the lift on $B_{j}$ to the set $b_{j+1}$. First observe that, for a point $\xi \in B_{j} \cap b_{j+1}$, there is a path $\beta$ joining $\xi$ to $y_{0}$ of length less than $5 \epsilon$. By considering the path $\tilde{\iota}_{j} \circ \beta$, it follows that $z=\tilde{\iota}_{j}(\xi)$ is contained in the ball of radius $8 \epsilon$ in $T_{x} F_{j}$. Hence by Lemma 13.1 we can construct a map $\exp _{z}^{-1}: \iota\left(b_{j+1}\right) \rightarrow B(0,10 \epsilon)$ which is a local inverse for the exponential map and so that $\exp _{z}^{-1}(\iota(\xi))=z$. We define $\tilde{\iota}_{j+1}$ on $b_{j+1}$ as $\exp _{z}^{-1} \circ \iota$. Note that this agrees with the previous definition of $\xi$. By the inverse function theorem, for each $y_{1} \in B_{j} \cap b_{j+1}$, the inverse image under the exponential map in $B(0,10 \epsilon)$ of $y_{1}$ is a discrete set. As $B_{j} \cap b_{j+1}$ is connected and both $\exp _{z}^{-1} \circ \iota$ and $\tilde{\iota}_{j}$ give lifts of $\iota$ on $B_{j} \cap b_{j+1}$ of the exponential map that agree at the point $\xi$, it follows that $\exp _{z}^{-1} \circ \iota$ agrees with $\tilde{\iota}_{j}$ on $B_{j} \cap b_{j+1}$. It follows that we have a well-defined extension $\tilde{\iota}_{j+1}$ of $\tilde{\iota}_{j}$. Proceeding inductively we obtain a lift $\tilde{\iota}$ as claimed.

We can ensure that $y$ lifts to the origin by picking a path $\alpha$ from $y$ to $y_{0}$ of length at most $3 \epsilon$. A simpler variation of the above argument gives a lift of this path to a path $\tilde{\alpha}$ beginning at the origin and ending at some point $z_{0}$. We proceed as before with $\tilde{\iota}\left(y_{0}\right)=z_{0}$.

\section{ACKNOWLEDGEMENTS}

The authors would like to thank Valentino Tosatti for helpful comments.

\section{REFERENCES}

1. W. K. Allard On the first variation of a varifold, Ann. of Math. (2) 95 (1972), 417-491. MR 0307015 (46:6136)

2. M. T. Anderson Curvature estimates and compactness theorems for minimal surfaces in 3manifolds, Ann. Sci. Éc. Norm. Super. IV. Ser. 18 (1985), 89-105. MR803196 (87e:53098)

3. H.I. Choi, R. Schoen The space of minimal embeddings of a surface into a three-dimensional manifold of positive Ricci curvature, Invent. Math. 81 (1985), 387-394. MR807063(87a:58040)

4. T. H. Colding, W. P. Minicozzi II Convergence of embedded minimal surfaces without area bounds in three-manifolds, C. R. Acad. Sci. Paris Sér. I Math. 327 (1998), no. 8, 765-770. MR.1659982 (2000i:58022)

5. M. Gromov Pseudoholomorphic curves in symplectic manifolds, Invent. Math. 82 (1985), no. 2, 307-347. MR809718 (87j:53053) 
6. J. Hass, G. P. Scott The existence of least area surfaces in 3-manifolds, Trans. Amer. Math. Soc. 310 (1988), no. 1, 87-114. MR965747 (90c:53022)

7. C. Hummel Gromov's compactness theorem for pseudo-holomorphic curves, Progress in Mathematics, 151, Birkhäuser Verlag, Basel, 1997. MR.1451624 (98k:58032)

8. J. Milnor, Morse theory, Annals of Mathematics Studies, No. 51, Princeton University Press, Princeton, N.J., 1963. MR.0163331 (29:634)

9. S. B. Myers Connections between differential geometry and topology I: Simply connected surfaces, Duke Math. J. 1 (1935), no. 3, 376-391. MR1545884

10. S. B. Myers Connections between differential geometry and topology II: Closed surfaces, Duke Math. J. 2 (1936), no. 1, 95-102. MR 1545908

11. P. Topping Relating diameter and mean curvature for submanifolds of Euclidean space, Comment. Math. Helv. 83 (2008), no. 3, 539-546. MR2410779 (2009b:53100)

12. B. White Curvature estimates and compactness theorems in 3-manifolds for surfaces that are stationary for parametric elliptic functionals, Invent. Math. 88 (1987), no. 2, 243-256. MR:880951 (88g:58037)

13. J. G. Wolfson Gromov's compactness of pseudo-holomorphic curves and symplectic geometry, J. Differential Geom. 28 (1988), no. 3, 383-405. MR965221 (89m:53058)

Department of Mathematics, Indian Institute of Science, Bangalore 560012, India

E-mail address: gadgil@math.iisc.ernet.in

Department of Mathematics, Indian Institute of Science, Bangalore 560012, India

E-mail address: harish@math.iisc.ernet.in 